\title{
The Guandimiao Bone Assemblage (and What it Says about the Shang Economy)
}

\author{
$\mathbf{\Delta} \cdot \mathbf{\Delta} \cdot \mathbf{\Lambda}$ \\ Hou Yanfeng, Roderick Campbell, Li Zhipeng, Zhang Yan, \\ Li Suting, and He Yuling
}

\begin{abstract}
The Anyang period village of Guandimiao - the only nearly completely excavated village in China of its time-is crucially important for understanding nonurban, nonelites in North China at the end of the second millennium B.c.E. Guandimiao was a small village that specialized in ceramic production, which indicates a hitherto unsuspected degree of economic specialization and integration in the Shang countryside. One line of evidence supporting an argument for economic integration between the urban center at Anyang and the countryside comes from the bone artifact assemblage recovered from the site. The Guandimiao bone artifact assemblage can be divided into four groups, each revealing a different path of production and distribution. One of these paths of production and distribution leads from this tiny village to Great Settlement Shang and its huge bone workshops some $200 \mathrm{~km}$ away. KeYwords: Shang, bone working, economy, zooarchaeology, China.
\end{abstract}

\section{INTRODUCTION}

Production studies have LONG BeEn used to ReVeal aspects of ancient political economies. From the early neo-evolutionary discourse concerning the putative relationship between specialized production and "states" (Brumfiel and Earle 1987; Costin 1991; Rice 1981) to more recent studies demonstrating the variety of modes by which early complex polities were provisioned (Flad 2007; Inomata 2001; Sinopoli 2003), the focus of most work has been on production for elites. Though archaeologists theorize ancient economies in terms of distribution and consumption in addition to production, in practice the connection between these three components (especially for nonelites) has been relatively understudied (Greene and Lindsay 2012). Studies of nonelite, rural consumption, production, and distribution in early complex polities are

Hou Yanfeng is an Assistant Researcher at the Archaeometry Laboratory, Henan Provincial Institute of Cultural Relics. Roderick Campbell is an Associate Professor at the Institute for the Study of the Ancient World, New York University. Li Zhipeng is an Associate Researcher at the Institute of Archaeology, Chinese Academy of Social Sciences. Zhang Yan is a doctoral candidate at the Institute for the Study of the Ancient World, New York University. Li Suting is a Researcher with the Henan Provincial Institute of Cultural Relics and Archaeology in China. He Yuling is an Associate Researcher at the Anyang Workstation, Institute of Archaeology, Chinese Academy of Social Sciences. 
vanishingly rare, yet extremely important. If economies can be seen as a skein of multidirectional networked interactions, then it is obvious that studying only one type of interaction, central node, and direction of connection is insufficient. Yet this has been exactly the case for research on the Shang polity centered at Anyang. What little work has been done on its political economy has focused on production centers at the capital, the extraction of resources such as metal and salt, and elite gifting (Campbell et al. 2011; Li 2003; Underhill and Fang 2004). Scholars have been quick to characterize the economy of the Shang polity as fundamentally "tributary" or "elite redistributive" (Chang 1980, 1989; Shelach-Lavi 2015; Underhill and Fang 2004; Yang and Ma 2010), but this determination has been more a projection from elite-biased texts and archaeological finds than a theory built from the solid foundation of systematic empirical research. This is why finding evidence in a tiny Shang village, not only of specialized ceramic production for regional consumption, but also of bone artifacts produced in the great workshops of the distant capital, is so remarkable. This study of Guandimiao bone working reveals patterns of distribution, consumption, and production that either have no relation to elites or indicate the flow of things not to, but from, the center.

Western archaeological literature has long held the belief that the Shang economy was underdeveloped outside of elite provisioning and intraelite redistribution (Chang 1975, 1980, 1989; Shelach-Lavi 2015). Moreover, the Chinese language literature has generally assumed that Shang village sites were simple agrarian hamlets or relic communes (Yang and Ma 2010; Zhongguo 2003). Contrary to both perspectives, Guandimiao and its worked bone assemblage reveal a hitherto unsuspected degree of political-economic integration in North China for this period.

The site of Guandimiao 关帝庙 in Xingyang 䓠阳, Henan was discovered in 2006 during salvage excavations undertaken as part of the Yangtze water diversion megaproject (nanshui beidiao 南水北调) (Fig. 1). Excavations continued until 2008. Remains dating to the Yangshao, Shang, western Zhou, eastern Zhou, Han, Tang, Song, and Qing dynasties were uncovered, but the most significant discovery was a nearly intact Anyang period village (Henan 2008, 2009) (Table 1). For this reason, the

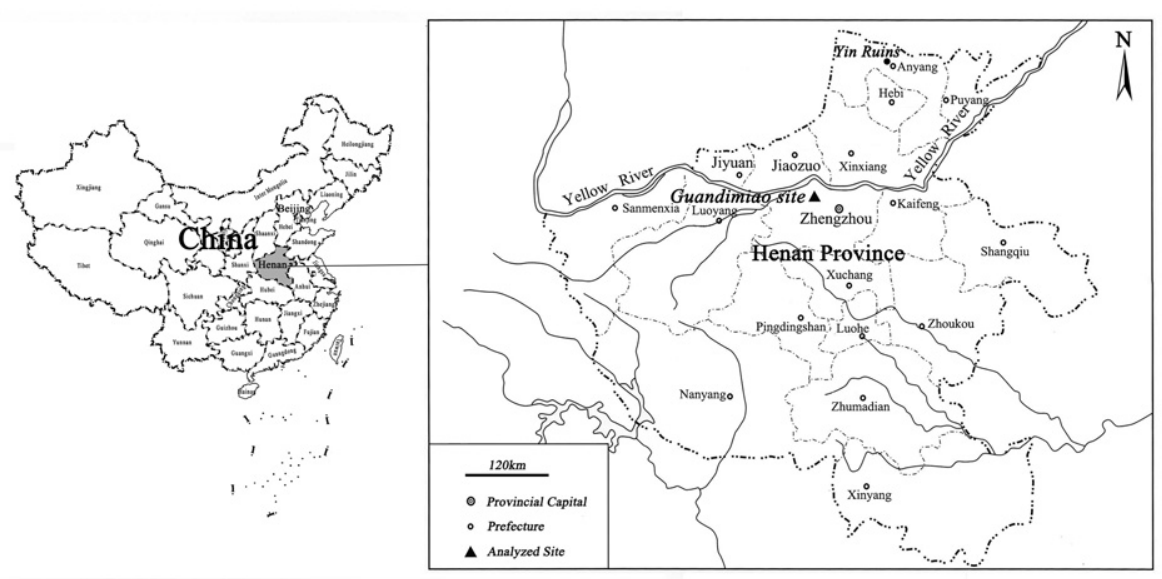

Fig. 1. Location of Guandimiao site in Xingyang, Henan, China. 
Table i. Chronology Associated with Guandimiao Site

\begin{tabular}{ll}
\hline PERIOD & \multicolumn{1}{c}{ DATES } \\
\hline Yangshao & $5000-3000$ B.C.E. \\
Anyang & $1250-1050$ B.C.E. \\
Western Zhou & $1050-771$ B.C.E. \\
Eastern Zhou & $770-221$ B.C.E. \\
Han & 206 B.C.E. - C.E. 220 \\
Tang & C.E. 618-907 \\
Song & C.E. $960-1279$ \\
Qing & C.E. $1644-1912$ \\
\hline
\end{tabular}

site was awarded Top Ten Discovery of 2007 by the State Administration of Cultural Heritage and Top Six Discovery of 2007 by the Academy of Social Sciences. Located in the region of the Erligang period (ca. 1600-1400 B.C.E.) center of Zhengzhou Shangcheng and the Xiaoshuangqiao-Huanbei period (1400- 1250 B.C.E.) center of Xiaoshuangqiao, Guandimiao was part of a network of small (and perhaps as-yetundiscovered large) Anyang period sites in what was once the capital area of the Erligang polity. During Anyang times, this area seems to have lost its metropolitan status, but nevertheless was likely part of the extended hinterland of Great Settlement Shang at Anyang, either as the nether edge of the Shang king's demesne, or, more likely, part of a network of sites under the sway of a local lord subordinate to the Shang king (Campbell 2009, 2014, 2018).

One of the most important discoveries made at Guandimiao was of a group of 23 updraft kilns, providing evidence of specialized ceramic production at the site (Henan 2008, 2009; Li et al. forthcoming). Given that only 22 small semi-subterranean houses and 269 tombs have been discovered for a village that was occupied for probably no more than 150 years (Anyang phase I-III: ca. 1250-1100 B.C.E.), the population likely never exceeded more than 100 people (Li et al. forthcoming). Given the close match between the number of houses recovered and the number of kilns, it is possible that each family unit had its own kiln. Nevertheless, the scale of production is far beyond that necessary for provisioning such a small population, which indicates that the Guandimiao village specialized in pottery production. Given the differences between Guandimiao and Anyang ceramics and the distances involved, it appears that the Guandimiao potters were not provisioning Great Settlement Shang with their ceramics. The most likely explanation is that the production was for regional consumption. The rural economy would, therefore, have been far more specialized and integrated than hitherto suspected. Whether this production occurred in a managed, centralized, redistributive economy or was for local markets or was distributed by itinerant traders remains an important question for future research.

The bone artifact assemblage recovered from Guandimiao reveals things about the site, and its economic connections are just as surprising and significant as the evidence of ceramic production in the village. We argue that the recovered bone artifacts can be divided into four groups along a continuum of specialization, skill, and labor (Choyke 1997): (I) expedient tools produced on-demand by unskilled labor; (II) low-tomoderate skill, low-labor tools produced locally with few or no specialized tools (perhaps by a part-time craftsperson); (III) oracle-bones laboriously produced by a local 
Table 2. Guandimiao Worked Bone Groups

\begin{tabular}{|c|c|c|c|c|c|c|}
\hline GROUP & CHARACTER & $\mathrm{NISP} / \mathrm{MNA}^{\mathrm{a}}$ & MODIFICATION & SPECIALIZED TOOLS & ASSOCIATED ARTIFACT TYPE & MATERIAL \\
\hline I & Expedient & $14(8.6 \%) / 14(12.1 \%)$ & Almost none & None & Awl/perforators & Highly variable \\
\hline II & Low skill/low labor & $52(32.1 \%) / 43(37.1 \%)$ & Little & Some drilling & $\begin{array}{l}\text { Awl/perforators, knives, } \\
\text { spatulas, shovels }\end{array}$ & Variable \\
\hline III & Moderate skill/high labor & $68(42 \%) / 32(27.6 \%)$ & Moderate & Chiseling & Oracle-bones & Specific \\
\hline IV & High skill/high labor & $28(17.3 \%) / 27(23.3 \%)$ & Complete & Sawing, drilling & Hair pins, arrowheads & Specific \\
\hline Total NISP/MNA & & $162(100 \%) / 116(100.1 \%)^{\mathrm{b}}$ & & & & \\
\hline
\end{tabular}

${ }^{a}$ NISP, Number of Identified Specimens (i.e., worked bone fragments); MNA, Minimum Number of Artifacts.

$\mathrm{b}$ Total MNA greater than $100 \%$ due to rounding. 
specialist with specialized tools; and (IV) non-local products made by skilled craftspeople following standardized production steps and using specialized tools and selective, high-effort, high-quality materials (Table 2). The artifacts of the fourth category are identical in all respects to those mass produced at the large-scale bone workshop of Tiesanlu, Anyang (Campbell et al. 2011).

\section{GUANDIMIAO WORKED BONE}

The raw materials for bone working are obviously derived from the animal economy more generally. Although more than three-quarters of the original village (over $20,000 \mathrm{~m}^{2}$ ) was excavated at Guandimiao, the zooarchaeological assemblage was relatively impoverished, yielding something like one-tenth of the animal bone recovered from a similar-sized excavation of the residential area of Xiaomintun, Anyang (Li et al. forthcoming). Deer and other wild taxa were rare in the assemblage at Guandimiao, but there was a relatively high quantity of cattle bone (Table 3). This is remarkable given the general belief that cattle were associated with elite ritual (Campbell et al. 2011; Li et al. 2011; Yuan and Flad 2005). Whatever the explanation for this phenomenon, from the perspective of Shang bone working, the relative abundance of cattle bone means that the villagers of Guandimiao did not lack highquality bone-working material.

\section{Group I: Expedient Bone Working}

The expedient tools found at Guandimiao were mostly awls or other perforators (Table 4). They can be characterized by a very low degree of bone modification, highly variable choice of raw materials, low degree of skill and labor, and the lack of specialized tools required to produce them (Fig. 2).

An obvious relationship exists between the nature of the tool and its production. Bone perforators can be made from any osseous material that naturally or through fragmentation has a pointed end. Very little effort or skill is required to make such artifacts.

A high degree of variability in the selection of raw materials is also characteristic of ad hoc or expedient tools. This follows naturally from their expedient nature: whatever can be used, is used. The relatively high frequency of antler (three pieces) is likely related to the fact that it was a relatively rare material at the site, though, due to its toughness, highly desirable for making heavy awls. That this valued material was not further processed is suggestive of the low level of local bone working.

The four artifacts shown in Figure 3 can be taken as representative of the expedient bone tools found at Guandimiao. One awl is a dog ulna; the only modification was that the distal end was ground into a point (Fig. 3A). A second awl is a medium mammal rib; again, the only modification was the grinding of one end into a point (Fig. 3B). On the other hand, a third awl made use of the natural point of an antler tine; it was only modified by being hacked off the antler rack and having a "handle" chopped into shape (Fig. 3C, Fig. 4A). The artifact shown in Figure 3D is one of the two bone spatulas that appear to have been produced from repurposed oracle-bones. This large mammal scapula was split rather than sawed, as can be seen from the lack of saw marks and the nonstraight sides. It was then shaped with a chisel (Fig. 4C), a tool commonly used in manufacturing oracle-bones at Guandimiao. The spatula was then ground smooth. 
Table 3. Guandimiao Zooarchaeological Assemblage

\begin{tabular}{lcc}
\hline TAXA & NISP $^{\mathrm{a}}$ & MNI $^{\mathrm{b}^{\mathrm{b}}}$ \\
\hline Cattle & $1629(37.96 \%)$ & $31(18.90 \%)$ \\
Pig & $1281(29.85 \%)$ & $62(37.80 \%)$ \\
Dog & $1144(26.66 \%)$ & $54(32.93 \%)$ \\
Sheep/goat & $159(3.71 \%)$ & $8(4.88 \%)$ \\
Deer & $71(1.65 \%)$ & $3(1.83 \%)$ \\
Rabbit & $5(0.12 \%)$ & $5(3.05 \%)$ \\
Horse & $2(0.05 \%)$ & $1(0.61 \%)$ \\
& & $164(100 \%)^{\mathrm{c}}$ \\
\hline
\end{tabular}

${ }^{a}$ Number of Identified Specimens.

${ }^{\mathrm{b}}$ Minimum Number of Individuals.

${ }^{\mathrm{c}}$ Figures based on preliminary analysis of data, may be slightly different in final zooarchaeological report.

Table 4. Expedient Artifact Types

\begin{tabular}{lcr}
\hline TYPE & NISP & MNA \\
\hline Spatula & 2 & 2 \\
Awl & 9 & 9 \\
Sickle/saw? & 1 & 1 \\
Debitage & 1 & 0 \\
Unclear & 2 & 2 \\
Total & $14^{\text {a }}$ & 14 \\
\hline
\end{tabular}

${ }^{\mathrm{a}}$ One large piece of antler showed evidence of having been the source of raw material for other artifacts (debitage), yet also having use-wear (unknown artifact type), and so was only counted once in the total.

While this artifact attests to a relatively high degree of effort to produce, if we are correct concerning its original purpose, the repurposing of the artifact only involved splitting a spatula-shaped portion off of the originally used oracle-bone's scapula and then grinding off the rough edges.

Fourteen expedient tools were recovered out of 116 artifacts (MNA) (Table 2). Given that the only criterion for production of these expedient bone perforators was access to suitably shaped pieces of bone and grinding stones or choppers, such tools were unlikely to have been acquired through exchange. The Group I class of artifacts was most likely produced on-site and ad hoc. Given the low degree of modification and the ease with which a tip might break off, leaving no evidence of its nature as a tool, it is also likely that there is a bias against the identification of artifacts in this class.

\section{Group II: Low Skill, Low-Labor, Unspecialized Bone Working}

The low-skill bone artifact group is distinguished from the expedient tools only in requiring a little more labor or skill or more specialized tools to produce. These 
Large mammal longbone

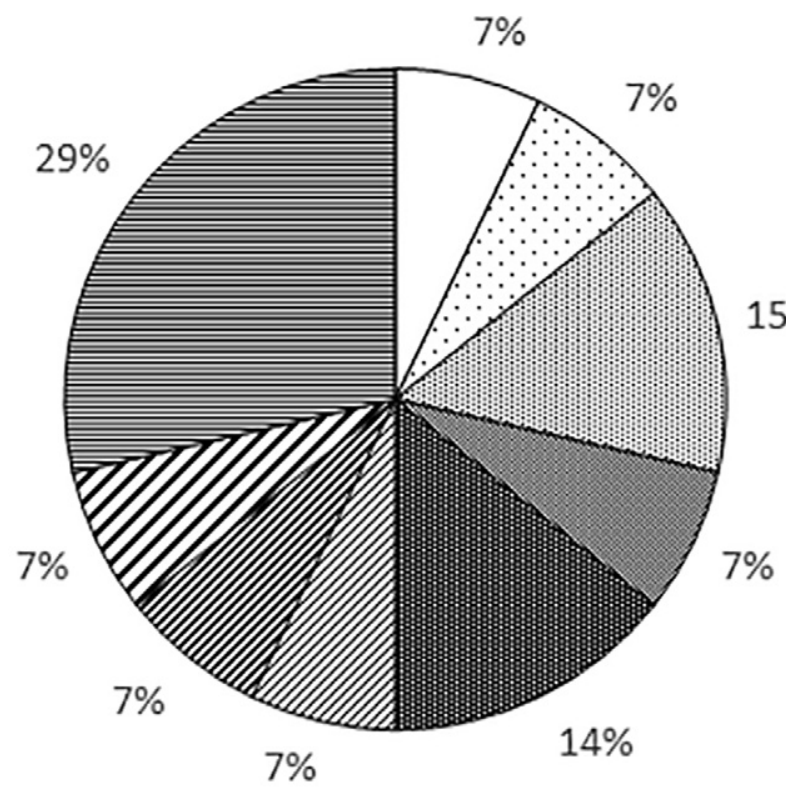

Large mammal rib

Large mammal scapula

Cattle ulna

$15 \%$

国 Med.-Large mammal rib

Medium mammal long bone

Medium mammal rib

EDog ulna

\section{目Deer antler}

Fig. 2 Group I: Expedient artifact raw materials by NISP.

artifacts include perforators, spatulas, spades, and knives (Table 5). The only evidence of production marks beyond splitting, chopping, or grinding are in a minority (30 percent) of artifacts bearing drill holes. Overall, this class of artifacts is characterized by a low degree of bone modification, skill, and labor. Though less variable than materials used for expedient bone working, the raw materials (mostly ribs) selected for Group II artifacts privileged ease of working and were characterized by a fit between the original shape of the bone and the shape of the desired artifact (Choyke 2013) (Fig. 5). Thus, thin flat spatulas, knives, and some perforators were produced from medium-to-large mammal ribs, other small perforators were whittled from medium-sized mammal long bones, and cattle mandibles were roughly chopped into spades.

The artifacts in Figure 6 are representative of the low-skill bone tool group. The artifact seen in Figure 6A is a thin perforator made from a large or medium-large mammal rib; it was split, then ground smooth and into a point at one end (see close-up in Fig. 7B). The Figure 6B artifact is a thin bone knife produced from a large mammal rib. The lack of straight lines and saw marks indicates that splitting and grinding were the chief methods used to manufacture, while the small drill hole and smoothly curved blade suggest that some care went into its production. On the other hand, this artifact did not require a great deal of modification from the original bone. Another artifact is a 


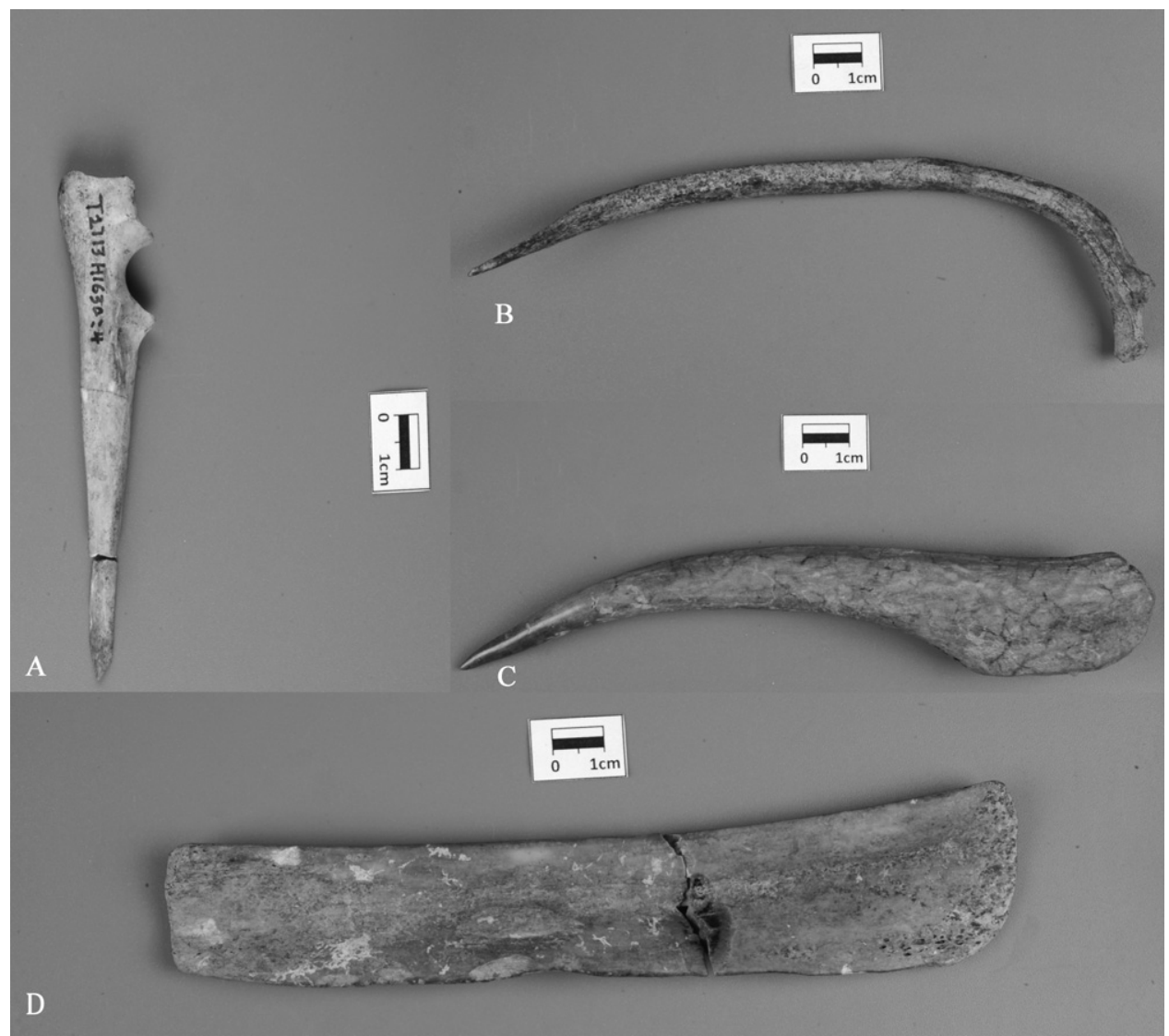

Fig. 3. Expedient tools: (A) dog ulna awl [HXYGT2713H1630:4]; (B) medium mammal rib awl [HXYGT4112H1090:3]; (C) antler tine awl [HXYGT3412H307:3]; (D) large mammal scapula spatula [HXYGT3216H125].

bone spatula made from a large mammal rib (Fig. 6C). Other than scoring and snapping off the ends (seen in Fig. 7D), the drilling of a hole, and some grinding, little labor was needed to produce this artifact. No specialized tools were required beyond a drill. The neatly scored and snapped rib ends and the drill hole nevertheless suggest that a level of skill and effort went into manufacturing this artifact beyond what was exhibited in the expedient tools.

Modified bone splinters are seen in Figure 6D and E. Based on the shape and size, the tool shown in Figure 6D was likely a needle or light awl of some sort. Some care was taken in tapering its long narrow point and shaping its narrow sides. The Figure $6 \mathrm{E}$ tool, however, may have been used to carve ceramics. It was shaped and had its edges ground into a form like a pointed guitar pick. A light-duty perforator produced from a medium mammal rib splinter is seen in Figure 6F. Other than the skill needed to split the tool into the desired shape (assuming it was not just chosen from among randomly produced splinters), the only thing that separates this artifact from the expedient tools is the neatly drilled hole in the handle. The artifact in Figure 6G is a damaged bone spade made from a cattle mandible. This artifact was chopped into shape rather than sawed 


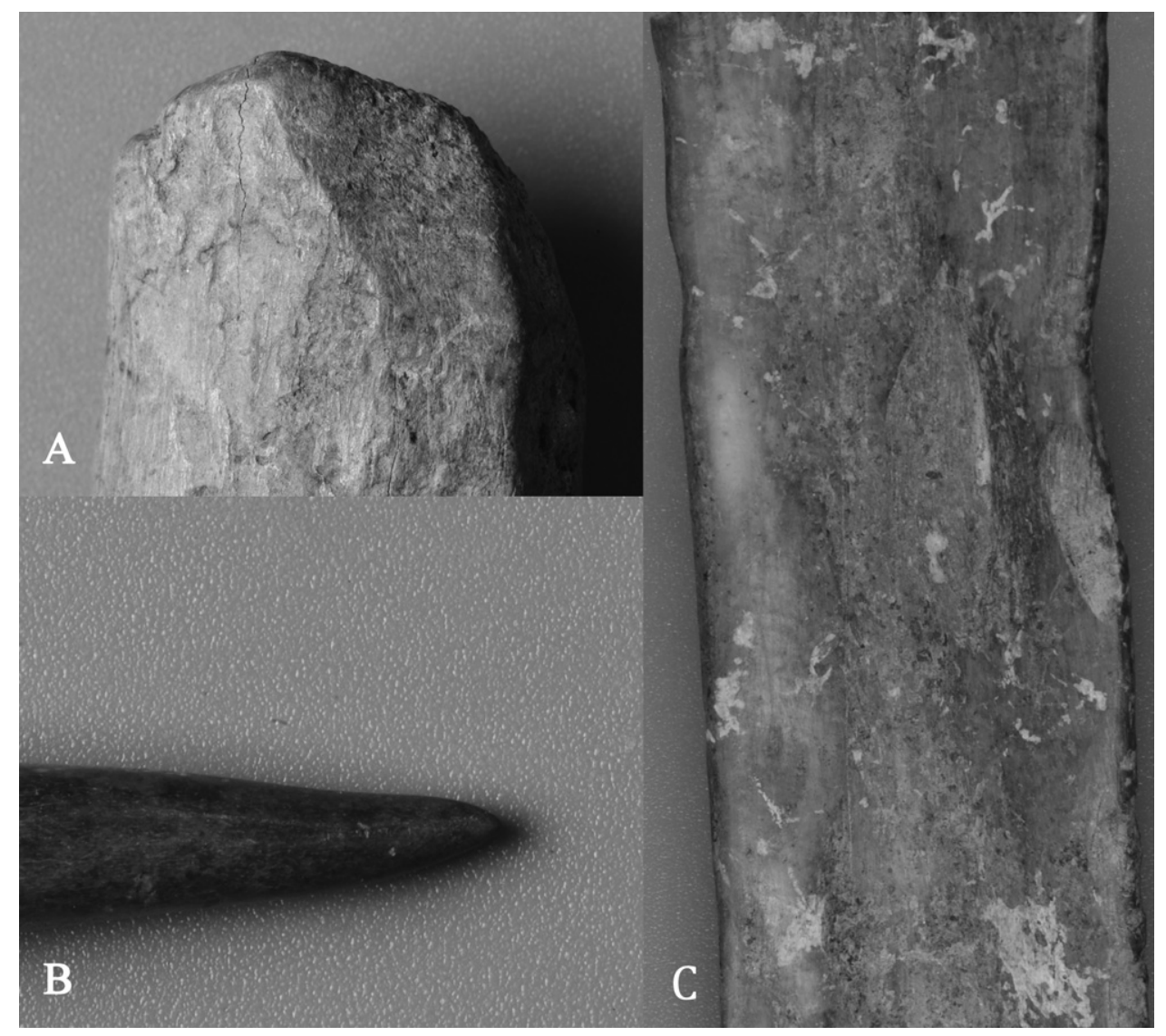

Fig. 4. Close-ups of expedient tools: (A) chop facets on handle and (B) polished natural antler tine, both from antler awl [HXYGT3412H307:3]; (C) close-up of chisel marks on scapula spatula [HXYGT3216H125].

Table 5. Low-Skill Artifact Types

\begin{tabular}{lrr}
\hline TYPE & NISP & MNA \\
\hline Spatula-shaped artifact & 22 & 20 \\
Knife-shaped artifact & 8 & 7 \\
Spade & 2 & 2 \\
Light awl/needle & 8 & 8 \\
Debitage & 4 & 0 \\
Unclear & 8 & 6 \\
Total & 52 & 43 \\
\hline
\end{tabular}


Large mammal rib

Cattle mandible

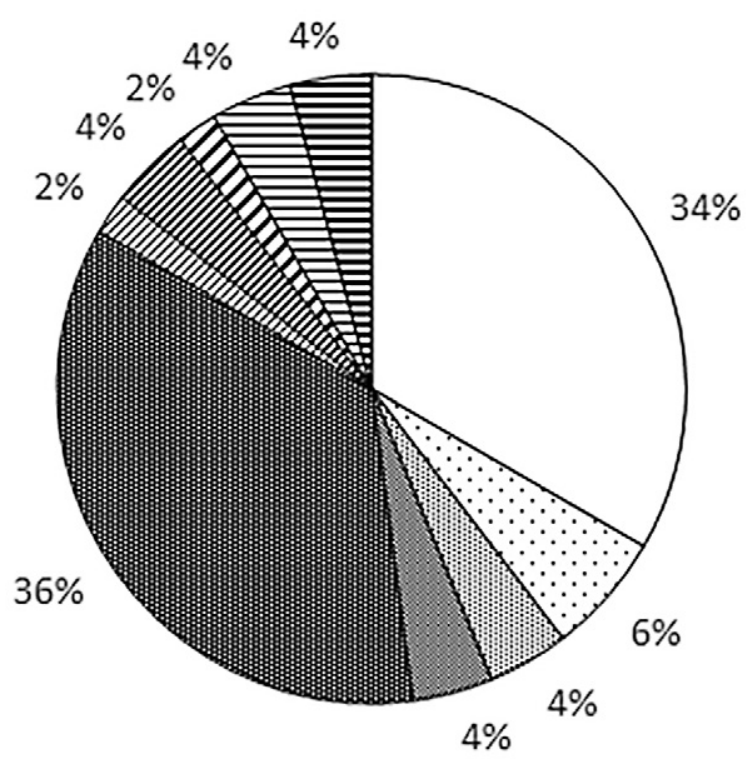

Cattle rib

Med.-Large mammal long-bone

国 Med.-Large mammal rib

ஐDeer antler

Deer metapodial

$\triangle$ Deer tibia

目Medium mammal
long-bone

目Medium mammal rib

Fig. 5 Group II: Low-skill/labor artifact raw materials by NISP.

(see close-up Fig. 7A). Little bone modification would have otherwise been necessary to produce the blade of this tool.

As noted above, Figure 7 shows closer views of the following production marks typical of Group II artifacts: cuts were made by chopping (Fig. 7A) or scoring and snapping (Fig. 7D) rather than sawing; points were often carefully tapered and ground (Fig. 7B); and holes were sometimes drilled (Fig. 7C). Taken together, these observations suggest that, while expedient tools might have been produced by anyone, the Group II artifacts were probably the work of people who at least had drills and grindstones and knowledge of bone working. Drilling holes for a string or thong also suggests their intentions to keep and reuse their tools. Still, Group II artifacts had relatively low requirements for production in terms of tools, skill, labor, and materials, which suggests that the artifacts in this group were unlikely to have been objects of long-distance exchange. Indeed, given the relative abundance of cattle bone at the site, there would have been little need to look elsewhere for the large mammal ribs that provided the raw materials used for most of these artifacts. The quantity of bone knife 


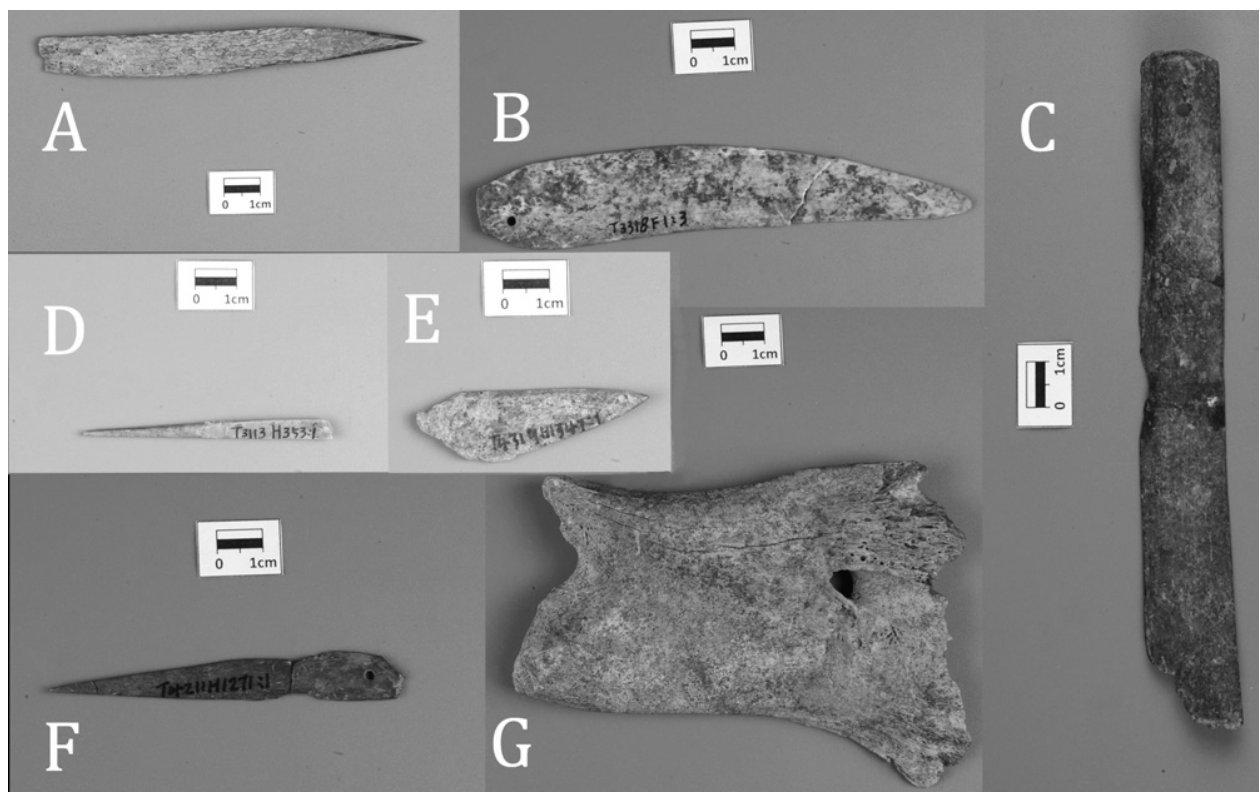

Fig. 6. Low-skill bone artifacts: (A) medium-large mammal rib perforator [HXYGT3918H738:1]; (B) large mammal rib knife with drill hole [HXYGT3318F1:3]; (C) large mammal rib spatula with drill hole [T4111H1061:1]; (D) bone sliver perforator [T3113H353:1]; (E) large mammal rib pointed tool [T4319H1347:1]; (F) medium mammal rib splinter perforator [HXYGT4211H1271:1]; (G) cattle mandible spade [HXYGT3114H421:1].

and spatula-shaped objects may well have been related to ceramic work, while the small amount of Group II debitage demonstrates at least some local production. Although cattle long bones are present in the zooarchaeological assemblage, these more difficult and time-consuming (but excellent) materials were apparently not worked at the site.

\section{Group III: Moderate-Skill, High-Labor, Specialized Bone Working}

The third category of Guandimiao worked bone is a highly specialized type of bone artifact production requiring moderate skill but high labor: oracle-bones. This type of bone working accounts for roughly 28 percent (32 MNA) of the worked artifacts recovered from the site. Compared to the low-skill expedient tools, oracle-bones are characterized by a relatively high degree of labor (especially for the scapulas), selection of specific materials (Fig. 8), some bone-working skill, and use of a specialized tool: the chisel (Table 6). Chisels were required for the production of the depressions in the bones, which were then heated until they cracked. Since none of the divination depressions were made by drilling (as seen in some Anyang oracle-bones), it seems the chisel was the chief tool used in preparing scapula and plastrons as divinatory surfaces at Guandimiao. This is most clearly seen in the cattle scapula oracle-bones as shown in Figure 9B, D and Figure 10, where the spine, part of the glenoid, and the caudal edge have been removed to create a flat surface. Splitting off the protruding bone without creating an undesired fracture required control, while the production of a flat surface 


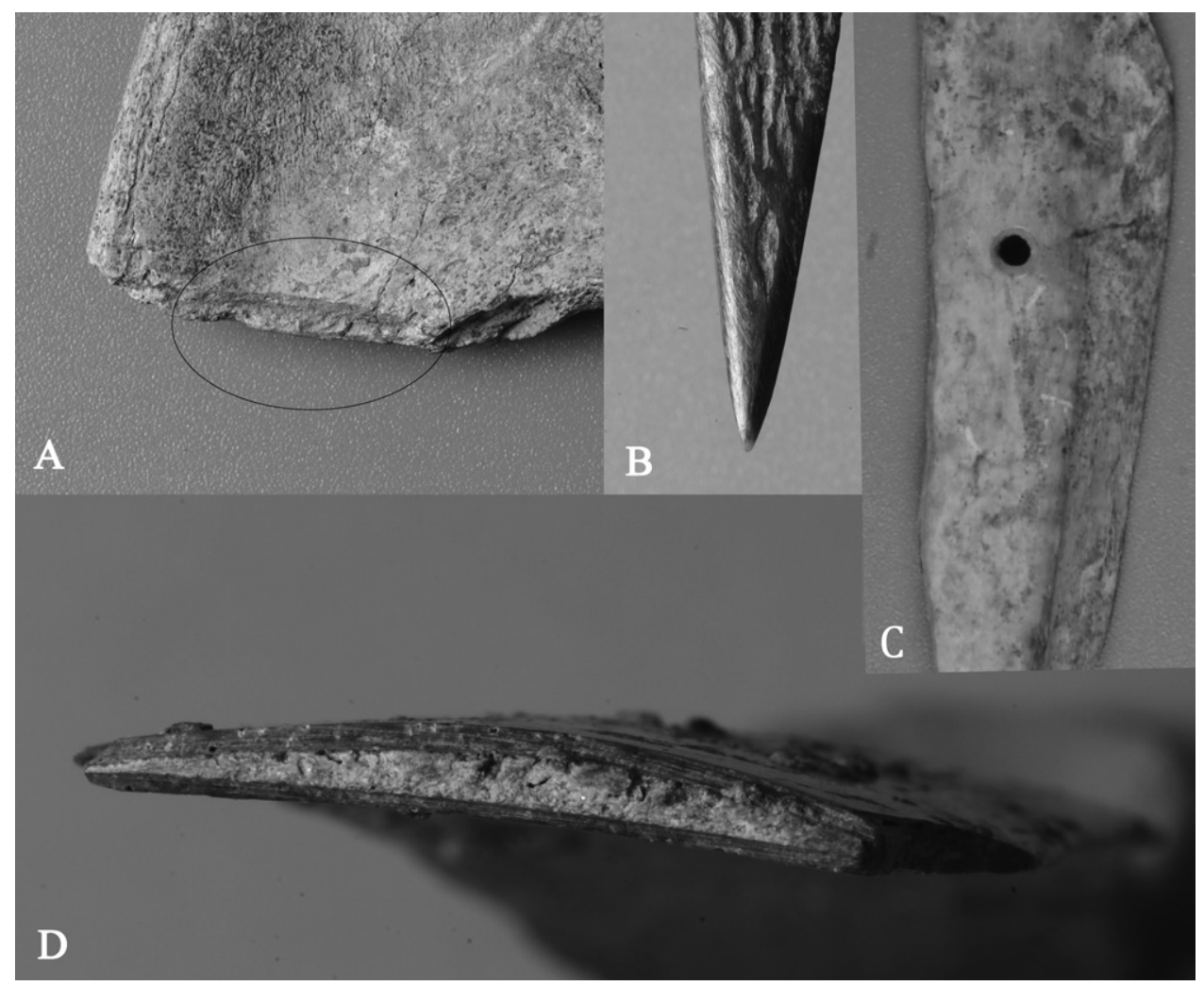

Fig. 7. Low-skill/specialization production marks: (A) chop marks [HXYGT3114H421:1]; (B) ground tip [HXYGT3918H738:1]; (C) drill hole [T3213H297:2]; (D) scored and snapped rib [HXYGT4019H1000:1].

necessitated tedious but careful chiseling, scraping, and grinding, all before the divinatory depressions could be chiseled into place and heat applied for divination.

The fragments in Figure 9 were chosen for their relative completeness, but are representative of the oracle-bone fragments recovered from Guandimiao. The oraclebone in Figure 9A is a turtle half-carapace fragment with eight chiseled and burned divination depressions on the inner side. The lack of straight edges and telltale striations on the bone suggest that no saw was used in production. Relatively little bone modification was necessary in its preparation beyond the removal of the dermal scutes and the chiseling of the divinatory depressions. The opposite is true of the bovine scapula (Fig. 9B, C, D). The not-quite-straight edges and lack of saw striations on one oracle-bone suggest that this fragment was skillfully split and then ground rather than sawed (Fig. 9C). The eight visible divinatory depressions were then carefully chiseled and cracked with heat. The oracle-bone in Figure $9 \mathrm{~B}$ is a bovine scapula with the entire dorsal side apparently split off before the spongy bone was gouged out with a chisel; it was then roughly ground flat before divination pits were chiseled into place and the bone was cracked. The oracle-bone in Figure 9D appears to have been made with more care and effort than the one in Figure 9B. The glenoid, spine, and caudal edge were neatly removed and the divinatory areas chiseled and then ground into a flat 
Cattle scapula

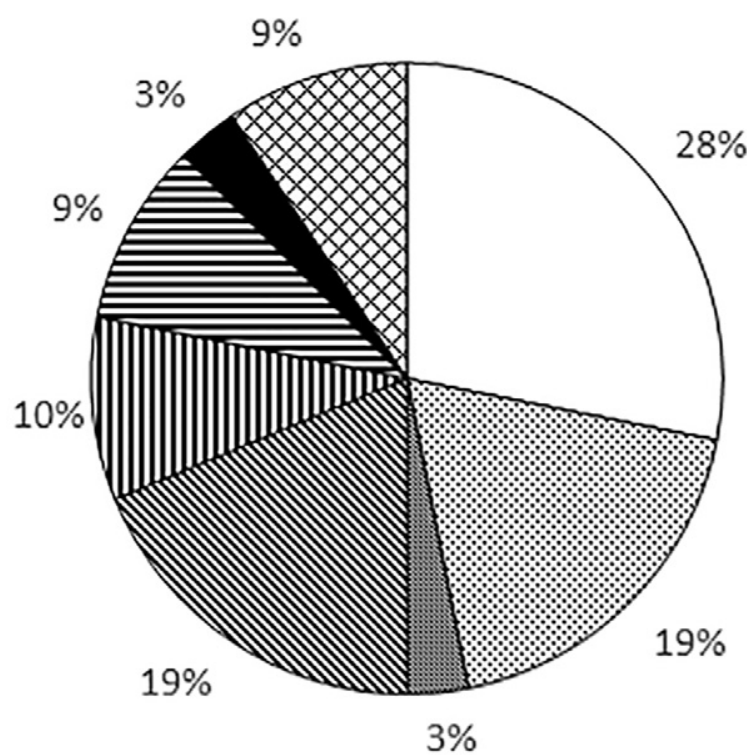

- $\mathrm{O}$ Large mammal

scapula

Med.-Large

mammal scapula

$\mathbb{\$}$ Turtle plastron

m Turtle carapace

Cattle pelvis

Horse pelvis

Q Large mammal

pelvis

Fig. 8. Group III: Oracle-bone raw materials by MNA.

Table 6. Guandimiao Oracle-bone Production and Use

\begin{tabular}{lrrrc}
\hline MATERIAL & NISP TOTAL & USED & UNUSED? & TOOL MARKS \\
\hline Large mammal (including cattle) scapula & 39 & 33 & 6 & $39{\text { chisel, } 3 \text { saw }^{\mathrm{a}}}^{\text {L }}$ \\
Large-Med mammal & 2 & 2 & 0 & 2 chisel \\
Turtle plastrons & 11 & 11 & 0 & 11 chisel \\
Turtle carapace & 9 & 5 & 4 & 7 chisel \\
Large mammal (including horse and cattle) pelvis & 7 & 4 & 3 & 7 chisel \\
Total & 68 & 55 & 13 & $66{\text { chisel, } 3 \text { saw }^{\mathrm{a}}}^{\text {Percentages }}$ \\
\hline
\end{tabular}

${ }^{a}$ Three fragments appear to have been cut with metal saws. Additional differences from the rest of the assemblage (i.e., divination cavities, care of production) suggest they may be Zhou period intrusions. One of these fragments was debitage from scapula modification for oracle-bone production.

surface. Neatly removing most of the dorsal side would have been more easily done with a saw than a chisel, and the apparently straight lines suggest the former tool could have been used; however, closer inspection reveals the straight edge was produced with a chisel after all (Fig. 10). 


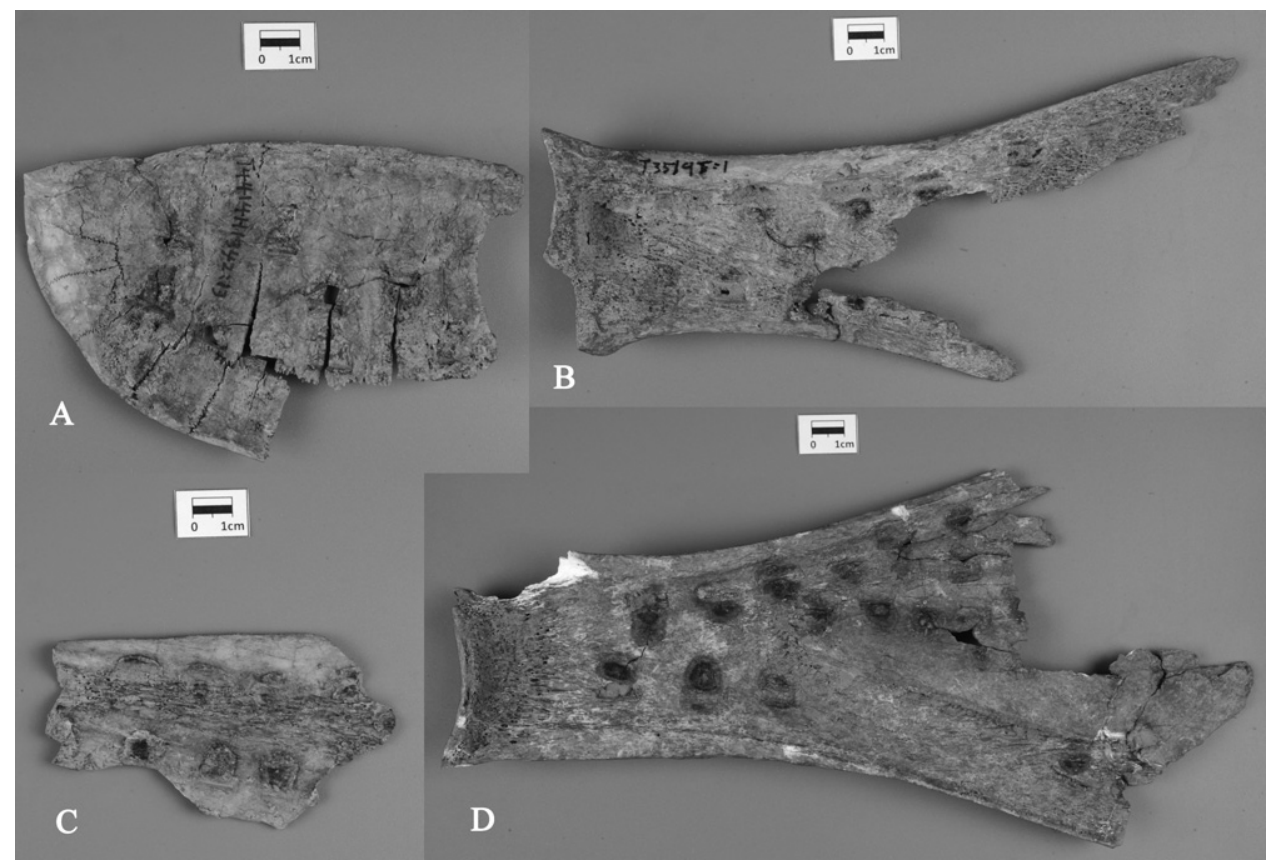

Fig. 9. Oracle-bones from Guandimiao: (A) turtle carapace [HXYGT4414H1342:13b]; (B) cattle scapula [HXYGT3519J7:1]; (C) large mammal scapula [HXYGT3114H421]; (D) cattle scapula [HXYGT3220H577:1].

While oracle-bones can be distinguished from low-skill and expedient artifacts in terms of the labor invested (especially in working scapulas) and specialized skills and tools (chisels) required for production, we postulate that they were also local products. As discussed below, they can be distinguished from oracle-bones produced at Anyang by their lack of saw marks. The presence of semi-prepared oracle-bone materials provides additional evidence of local production (see Flad 2008 for a discussion of diviners as specialist producers). For example, the bone articulations on the interior of the turtle carapace shown in Figure 11 are not present; they were possibly intentionally removed, but the divinatory depressions were not yet chiseled. Additional lines of evidence for local production include the following: the relatively high percentage (19 percent) of bones partially prepared, but with no signs of use (Table 6); the close match between numbers of oracle-bone scapula MNA (16) to cattle MNI (31), demonstrating that Guandimiao oracle-bones could easily have come from local cattle; the use of a relatively wide range of materials, even pelvic bones (Fig. 8); and the absence of cattle scapula not used for divination. All of these observations taken together suggest that local diviners used specialized skills and tools to produce oraclebones, but nothing else, and all from local osseous materials.

While a full comparative study of the Guandimiao oracle-bones as Shang divinatory materials will be published elsewhere, for the purposes of this article it is enough to note that the skilled, specialized, and laborious local bone working was different from Anyang oracle-bone production and separate from local production of bone tools. 


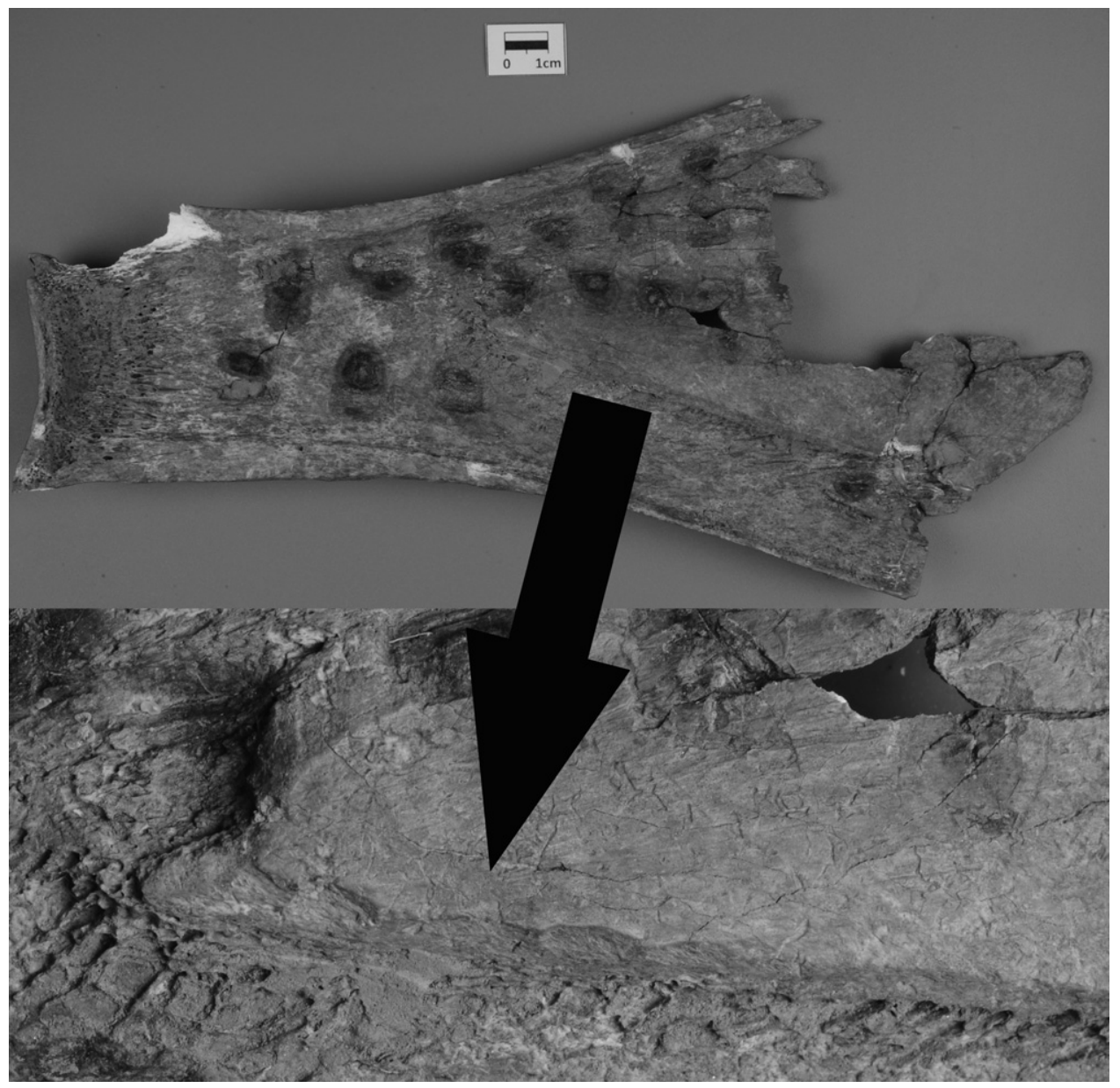

Fig. 10. Oracle-bone cattle scapula chisel marks [HXYGT3220H577:1].

\section{Group IV: High-Skill, High-Labor, Highly Specialized Bone Working}

The fourth category of bone artifacts recovered from Guandimiao is distinguished by complete modification of the original bone, standardized production steps, a high degree of labor and skill, the ubiquitous use of bronze saws, and selection of particular materials (Fig. 12). Almost all of these 27 artifacts $(23.3$ percent of the worked bone assemblage by MNA) are hairpins, but there were also two arrowheads (Table 7).

Examined closely, there are some glaring differences between Group IV artifacts and those in the other three groups. First is the use of high-quality, but labor-intensive materials such as large mammal long bones and antlers. While some antlers would have been available locally, there are no signs of antlers having been worked with local techniques (i.e., chopping, splitting, chiseling, or grinding) beyond the minimal preparation of expedient tools. The lack of worked cattle long-bone debitage is even more telling. Without saws, cutting off the articular ends and scoring and then splitting the bones is a laborious process, made more difficult by the necessity to grind down the 


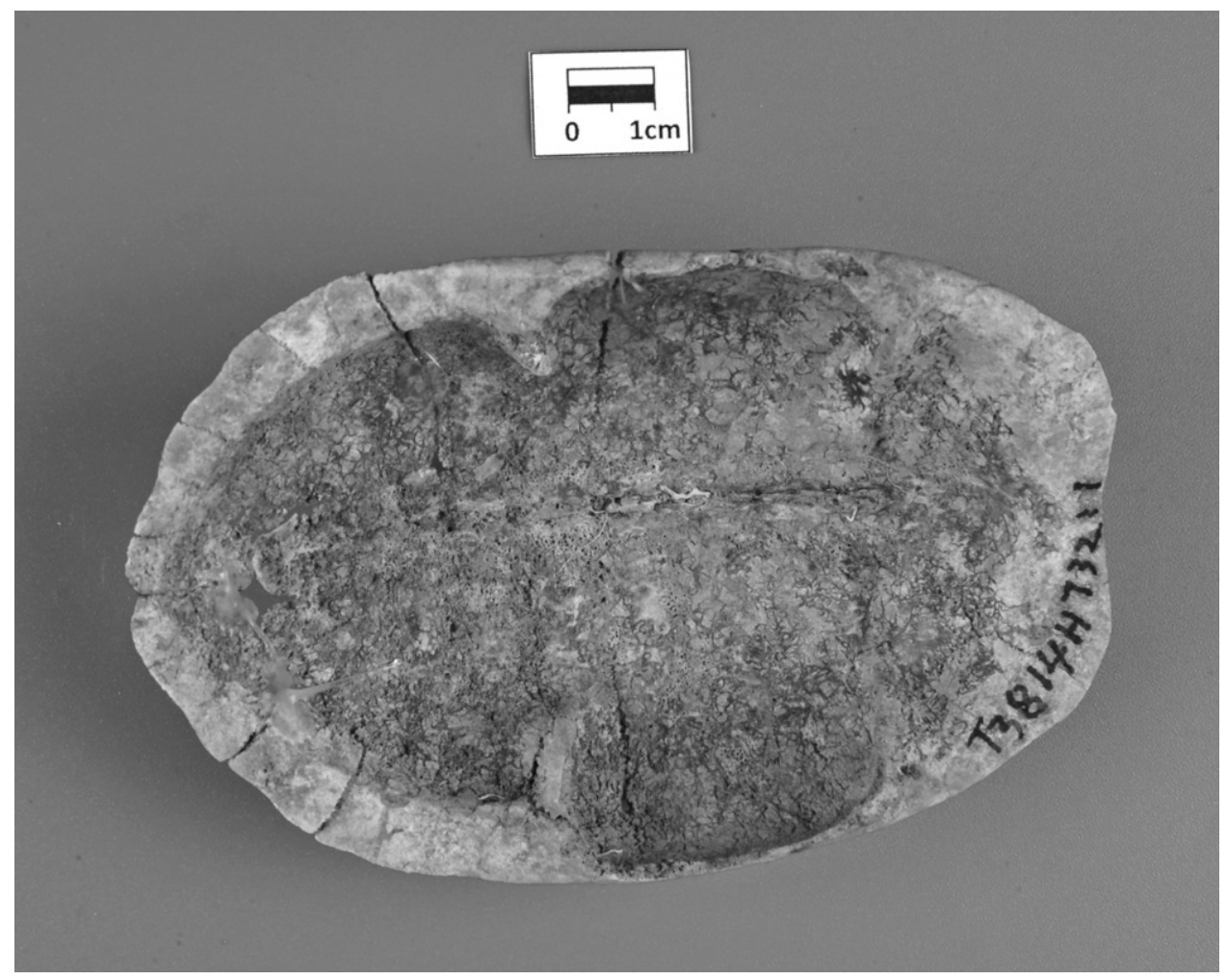

Fig. 11. Semi-prepared turtle carapace [HXYGT3814H732:1].

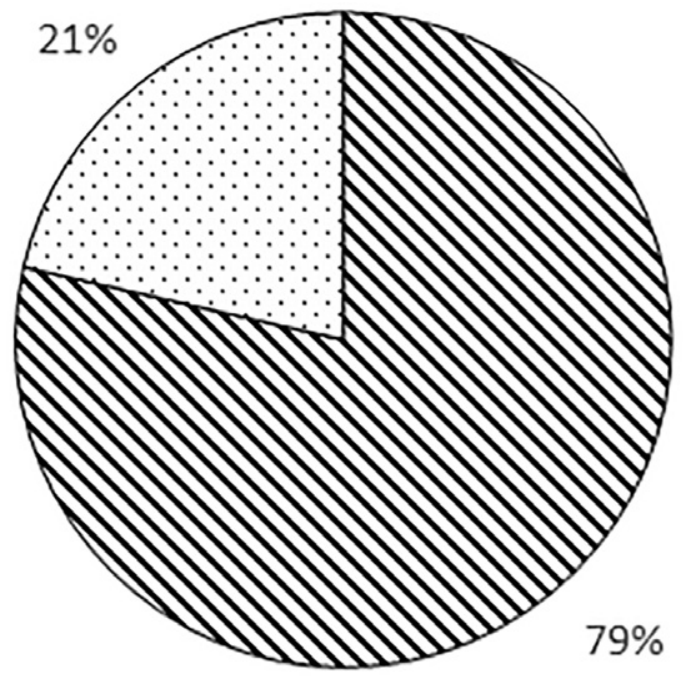

$\boldsymbol{\Delta}$ Large mammal longbone

$๑$ Deer antler

Fig. 12 Group IV: High-skill/labor bone-working materials by NISP. 
Table 7. High-Skill, Specialized Artifact Types

\begin{tabular}{lcr}
\hline TYPE & NUMBER & MNA \\
\hline Hairpin & 25 & 25 \\
Arrowhead & 2 & 2 \\
Debitage & 1 & 0
\end{tabular}

Total

resulting strips into spatulas, arrowheads, pins, or awls. These production steps also leave large quantities of debitage, usually many times the number of finished artifacts. Only a single fragment of neatly sawed debitage was recovered from Guandimiao; it is most likely a later intrusion. A second difference is the degree of skill, standardization, and effort that went into producing artifacts such as those seen in Figure 13. They are all nearly identical to artifacts recovered from Anyang, but differ from the other classes of artifacts found at Guandimiao. Finally, most Group IV artifacts show signs of having been produced with metal saws. If these had been local products, we would have expected them to be roughly split and ground, to have discovered many fragments of scored and split cattle long-bone debitage, or to have found other evidence of saw use at the site.

The first two bone artifacts (Fig. 13A, B) are decorative pins from large mammal long bones; they have been sawed, rasped, polished, and then perforated at one end and notched to be inserted into a separate pinhead. The artifact in Figure 13C is a plain hairpin, also sawed, rasped, and polished from a large mammal long bone. The next is a cylindrical-type antler arrowhead (Fig. 13D). The next two artifacts on the top row are "double-head nail" type hairpins (Fig. 13E, F). Despite the oval-shaped head, the straight-line striations demonstrate this design was executed with a saw (see Fig. 14A). The pins in the bottom row are all of the same abstract design, executed with a saw, and produced from large mammal long bones (Fig. 13G-L). As can be seen from the straight cuts and the striations left on the bone, they were first cut into performs, and then the basic design was cut into them with a saw (Fig. 14B, C) (Campbell et al. 2011). The sharp edges were then rasped off of the body in a diagonal motion, most likely also with a bronze saw; the teeth marks of the saw can still be seen on some of the pin bodies even after polishing (Fig. 14D).

The different lengths of the hairpins can be accounted for by breakage and reuse, as testified by the nonsymmetrical but nonetheless polished tips of the shorter pins (Fig. 13G and J are especially obvious examples). The standardization of length, style, production steps, tool marks, choice of materials, and degree of effort and skill required to make these artifacts all set them apart from the other three classes of worked bone at Guandimiao (Table 2).

THE ORIGINS OF GROUP IV BONE WORKING

Thus far we have presented evidence to support separating the bone artifacts found at Guandimiao into four groups according to the variables of raw material selection, bone modification, tool marks, skill, and labor (Table 2). The data presented in Table 2 makes 


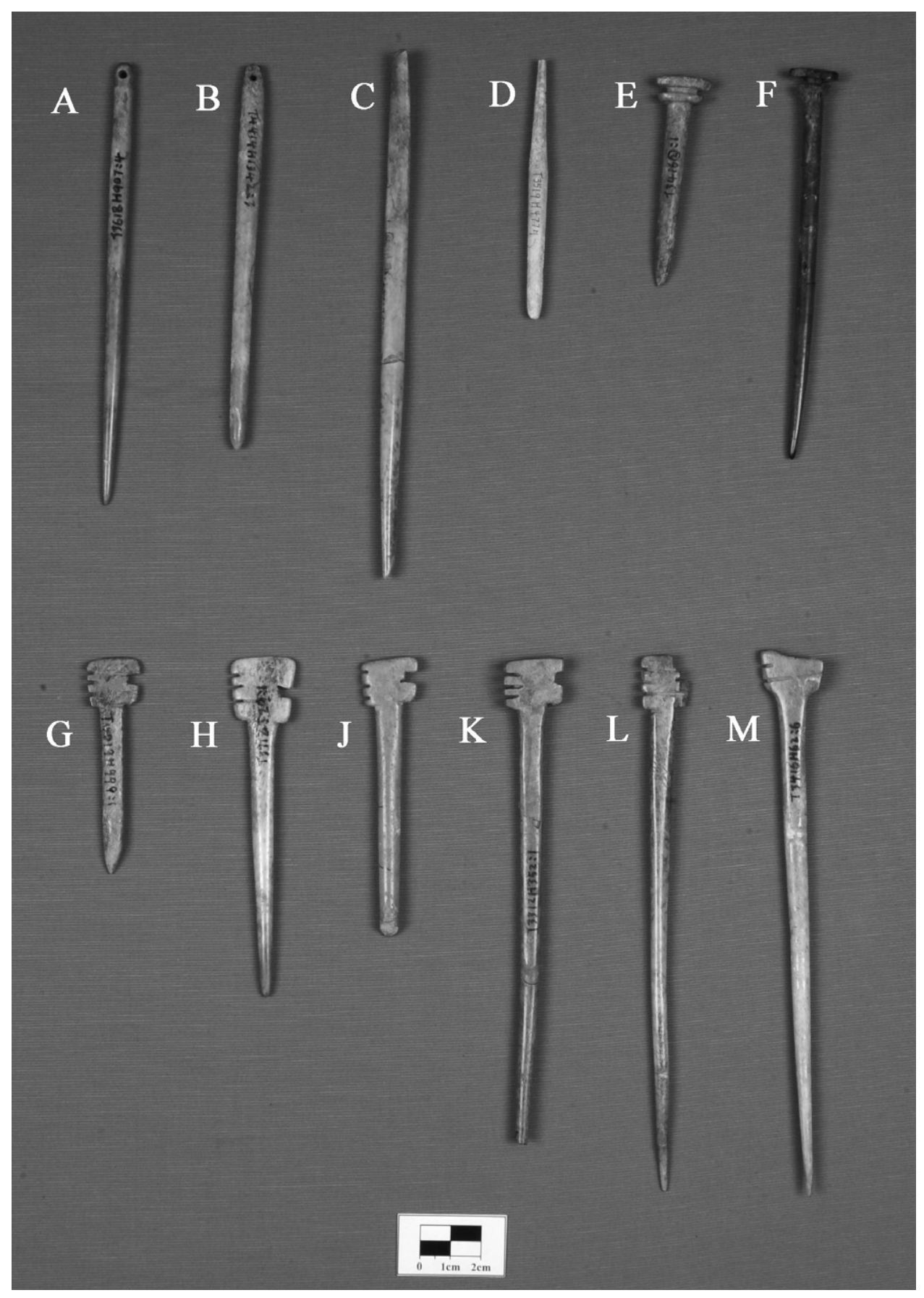

Fig. 13. High-skill/labor, specialized bone working: (A) pin with perforated head for attachment [HXYGT3618H907:4]; (B) perforated pin [HXYGT4414H1342:3]; (C) undecorated pin [HXYGT3313H10(2):1]; (D) cylindrical type arrowhead [HXYGT35519H477:1]; (E) double nail head hairpin [HXYGT3416(2):1]; (F) double nail head hairpin [HXYGT3413H7:1]; (G) abstract bird design pin [HXYGT4019H999:1]; (H) abstract bird design pin [HXYTG3718J29:1]; (I) abstract bird design pin [HXYGT4018H994:1]; (J) abstract bird design pin [HXYGT3312H352:1]; (K) abstract bird design pin [HXYGT4111H1059(3):2]; (L) abstract bird design pin [HXYGT3416H62:6]. 


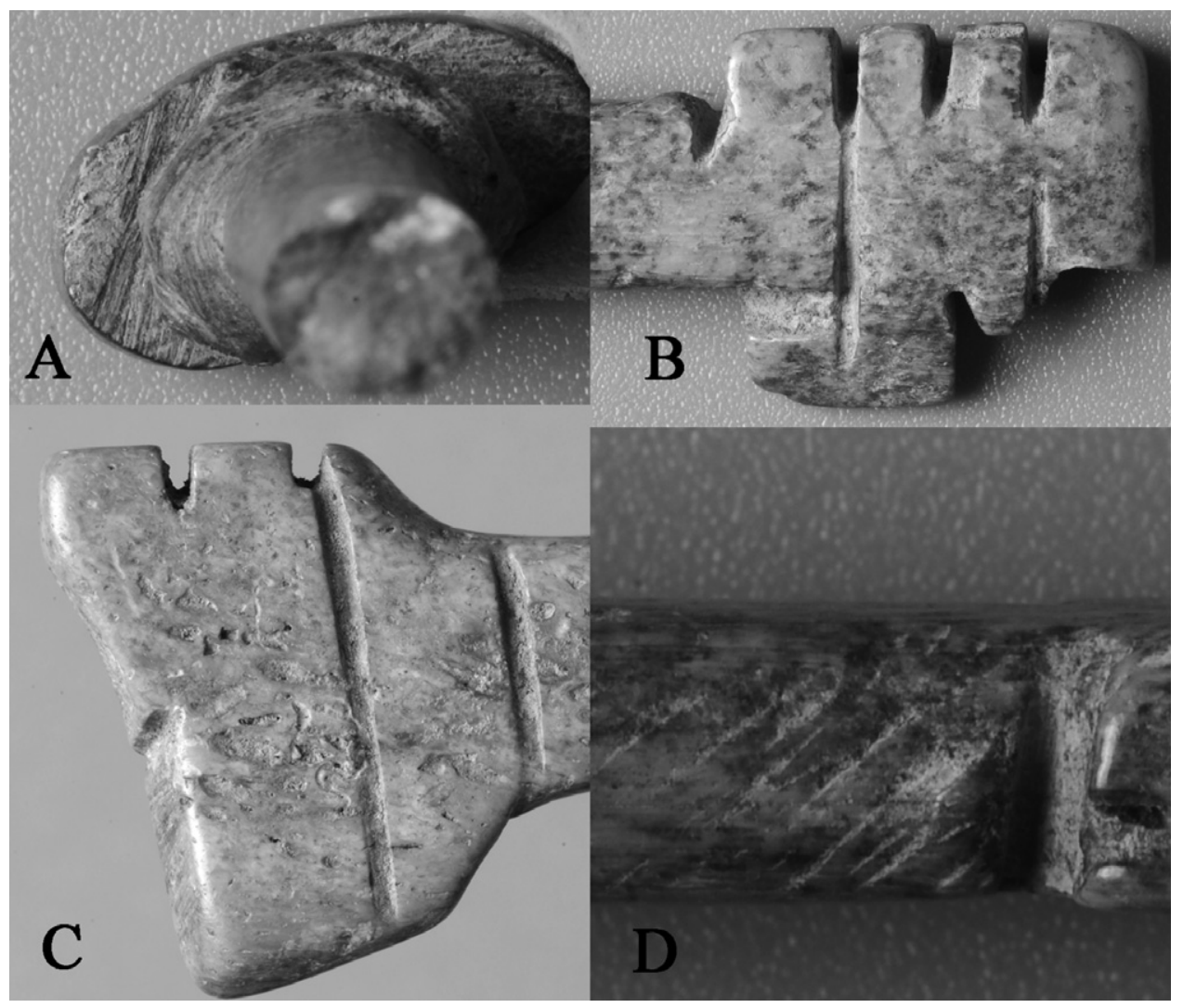

Fig. 14. Specialized bone workshop product tool marks found at Guandimiao: (A) double nail head pinhead with saw striations on head bottom [HXYGT3413H7:1]; (B) abstract design, one-piece pinhead saw cuts [HXYGT4111H1059(3):2]; (C) broken abstract design, one-piece pinhead showing saw striations on cut surface [HXYGT3416H62:6]; (D) abstract design, one-piece pin body near head with rasping teeth marks remaining after polishing [HXYGT4111H1059(3):2].

it apparent that while a majority of the non-oracular bone artifacts recovered from Guandimiao were of the low-skill, low-labor type argued to be of local manufacture, a significant percentage (23 percent) were of the high-skill, high-labor type, which we argue were produced by nonlocal craft specialists. Our argument against Group IV artifact production having taken place at Guandimiao is, first of all, the lack of worked cattle limb bone production waste, blanks, and half-finished artifacts that the manufacture of pins like those found at Guandimiao inevitably produces (Campbell et al. 2011; Li et al. 2011). Secondly, the use of specialized tools as well as labor- and skillintensive processes for only two artifact types logically suggests a mode of production that differs from that which produced the other artifact types in the assemblage. In other words, if the spades, awls, spatulas, and knives recovered from Guandimiao were made by the same craftspeople that made the hairpins and arrowheads, why did they fail to use the same tools, techniques, and materials?

Moreover, a contemporaneous bone artifact assemblage is available for comparison. It starkly demonstrates what awls, spatulas, and even oracle-bones look like when they 
are produced in a specialized, large-scale workshop context. Below, we systematically compare and contrast the bone artifact assemblage recovered from Guandimiao with artifacts from the bone workshop at Tiesanlu, Anyang (Campbell et al. 2011; Zhongguo 2015).

\section{Preparation of Material}

A clear difference can be seen between worked bone fragments recovered from Guandimiao and those recovered from Tiesanlu, beginning with the preparation of material, that is, the cutting of bone into smaller pieces for working (Fig. 15). The Guandimiao worked rib fragments are all either carefully scored and snapped to prevent undesired fragmentation (Fig. 15D, E) or they are chopped (Fig. 15A), risking damage to the thin cortical bone and the creation of a ragged edge. Rib working at Tiesanlu, however, was not only restricted to large mammal ribs, but uniformly involved sawing (Fig. 15F). This is a much more efficient procedure allowing the same control as scoring and snapping; it is also achieved with less effort. A comparison with worked antler shows the same pattern: while Guandimiao antler was hacked into pieces (Fig. 15B, C), Tiesanlu antler was neatly sawed into smaller pieces (Fig. 15G). In this instance, the chief advantage of a saw over a chopping tool is control rather than speed. Comparing worked mandibles from Tiesanlu and Guandimiao reveals the same pattern: while the mandible spade in Figure $6 \mathrm{G}$ was chopped into shape (see also Fig. 7A), Tiesanlu mandibles were neatly sawed (Fig. 15H). These examples clearly demonstrate that there are fundamental differences between bone-working procedures and tools at Guandimiao and Tiesanlu, starting with the first step of bone material preparation. In other words, despite the clear superiority of sawing over chopping or

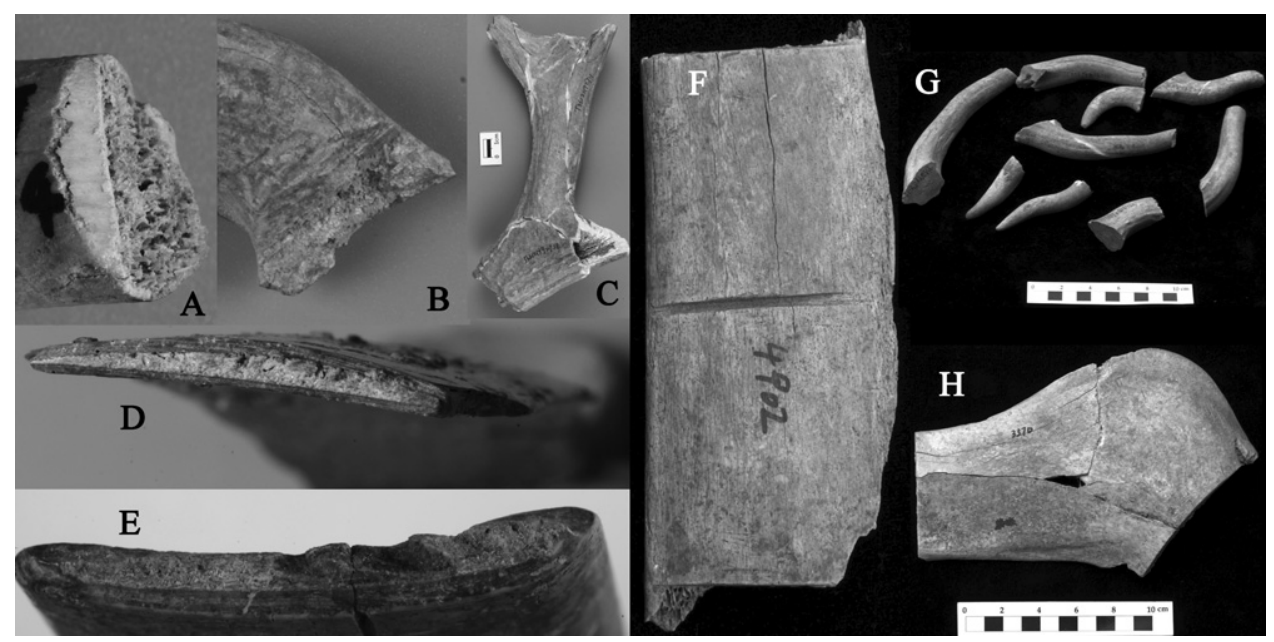

Fig. 15. Comparison of bone preparations from Guandimiao (left A-E) and Tiesanlu, Anyang (right F-H): (A) rib with chopped end [HXYGT3315H11:4]; (B) antler with chop marks [HXYGT3612H927:1]; (C) antler [HXYGT3612H927:1]; (D and E) scored and snapped rib ends [HXYGT4019H1000:1]; (F) rib pre-form waster with saw marks [ATST5:4902H7]; (G) sawed antler materials [ATST5]; (H) sawed cattle mandible pre-form [ATST5:3570H21]. 
scoring and snapping for the preparation of bone-working materials, those preparing osseous materials at Guandimiao or producing low-skill, low-labor artifacts did not use bronze saws. They probably did not possess such tools, since there is very little bronze at Guandimiao in general (Henan 2008, 2009; Li et al. forthcoming).

As noted above, the vast majority (97 percent) of oracle-bones produced at Guandimiao were made without the aid of saws. This suggests that even local highskill, high-labor, specialized bone workers lacked the most advanced tools available at central sites such as Great Settlement Shang. The difference is clearly on display in Figure 16, which compares the articular end of an oracle-bone scapula excavated from

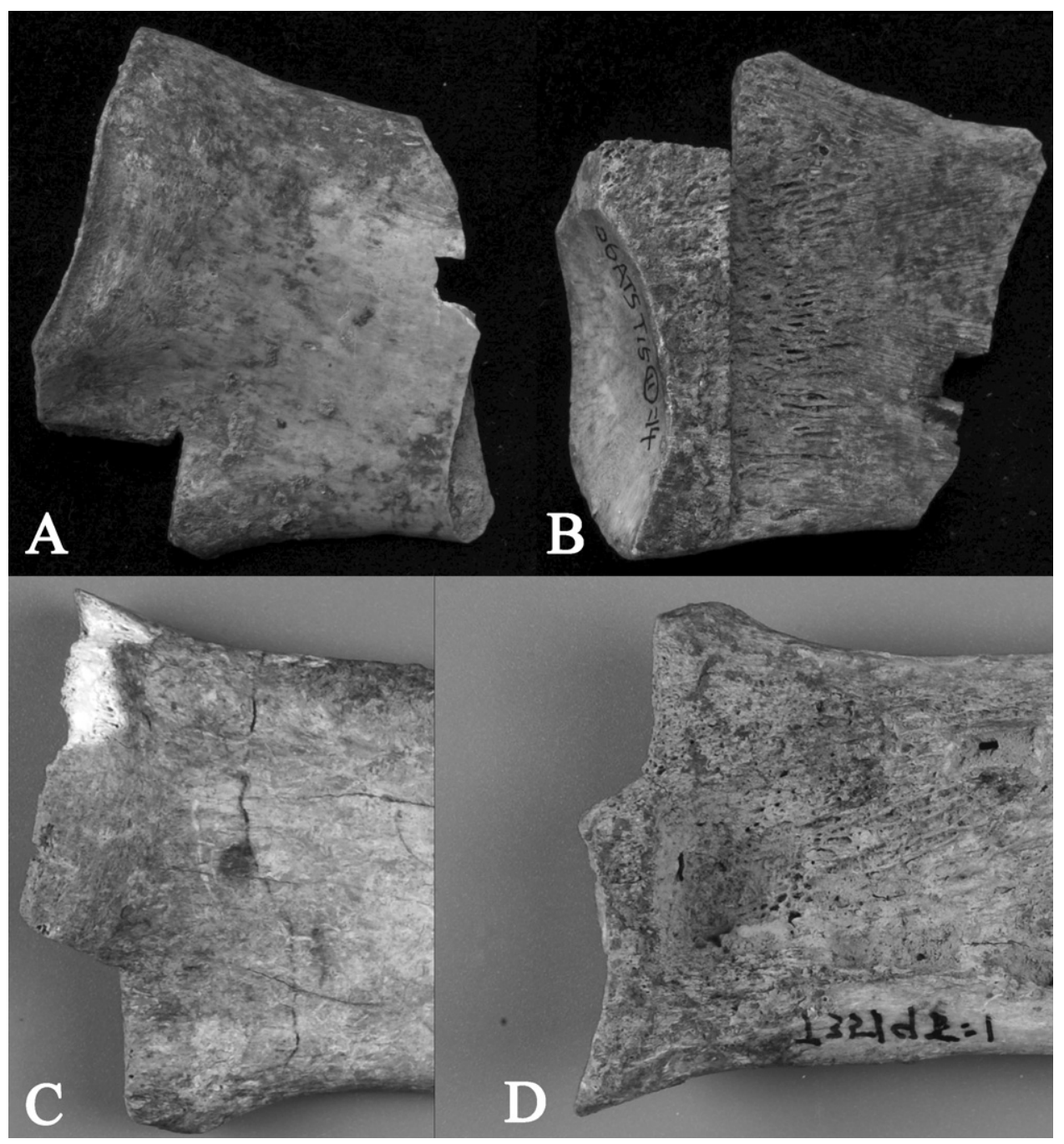

Fig. 16. Comparison of oracle-bones from Tiesanlu, Anyang (top) and Guandimiao (bottom): Tiesanlu oracle-bone fragment, glenoid cavity, and neck [ATST15(11):14], (A) ventral side, (B) dorsal side; Guandimiao oracle-bone fragment, glenoid cavity, and neck [HXYGT3519J7:1], (C) ventral side, (D) dorsal side. 
Tiesanlu with the same part of an oracle-bone scapula from Guandimiao. While in both cases the caracoid process has been removed, the saw used on the Tiesanlu oraclebone produced straight edges, a right angle, and small saw overcut (Fig. 16A). Although an effort was made to carefully remove the caracoid process without cracking the bone in the Guandimiao scapula, the craftsperson nevertheless failed to produce completely straight edges or a right angle; in this case, caracoid removal involved a more laborious and less neat process of splitting and grinding rather than sawing (Fig. 16C). A comparison of the dorsal sides of each bone yields an even clearer distinction. While the perfectly flat faces, straight angles, and sawing striations of the Tiesanlu bone bear mute testimony to the use of a metal saw blade (Fig. 16B), the Guandimiao bone (Fig. 16D) displays not-quite-straight edges and a not-entirely-flat divinatory surface hollowed out with a chisel. While it is undoubtedly far more efficient (and the results are more regular) to produce a flat surface with a saw rather than a chisel, the craftspeople or diviners who produced the Guandimiao oracle-bones did not use saws, most likely because they did not have them. Instead, they laboriously produced cruder, less neat versions of metropolitan divinatory media without the aid of the specialized bone-working tools available in the capital.

\section{Specialized versus Nonspecialized Bone Working: Awls and Spatulas}

Comparing the awls and spatulas recovered from the same two sites further demonstrates that the low-skill, low-labor artifacts from Guandimiao were produced in a manner fundamentally different from artifacts produced at large-scale, specialized workshops such as Tiesanlu. While, as at Guandimiao, many of the spatulas produced at Tiesanlu were made from ribs (albeit sawed), large mammal limb bones such as the humerus and femur were also used to produce some of the Tiesanlu spatulas; these bones require a more labor-intensive process, but produce much sturdier spatulas. A comparison of a Guandimiao spatula (Fig. 6C) with a Tiesanlu spatula (Fig. 17A) reveals that the former is basically a scarcely modified rib, while the latter is a carefully sawed strip of large mammal long bone, with striations produced by the saw clearly visible on its unfinished edges.

The awls produced at Tiesanlu, moreover, provide an even starker distinction. Unlike the expedient tools excavated at Guandimiao, the awls produced at the Tiesanlu workshop were largely made from antler, a material especially suited to resisting shock and preferentially used in awls and arrowheads the world over (MacGregor 1985; MacGregor and Currey 1983). They were also highly modified: once a suitable piece of antler was cut from the rack, a long, pointed, rectangular prism pre-form was sawed into shape (Fig. 17B); the edges were then rasped smooth in a diagonal motion with a toothed tool, most likely a bronze saw (Fig. 17C). The result was a lightweight but sturdy tool, much stronger than either the Guandimiao dog ulna or medium-sized mammal rib awls (Fig. 3A, B), and lighter and more wieldy than the antler awls recovered from Guandimiao (Fig. 3C). Once again, the entire production process is distinguished by high-quality materials, high-labor input, and the ubiquitous use of metal saws at Tiesanlu, from the initial raw material preparation to the cutting of preforms and likely subsequent rasping of the edges before the final polishing stages (for those artifacts that were polished) (Campbell et al. 2011).

The Group IV bone artifacts from Guandimiao clearly cluster with the Tiesanlu production pattern while differing from the other three locally produced groups. To 


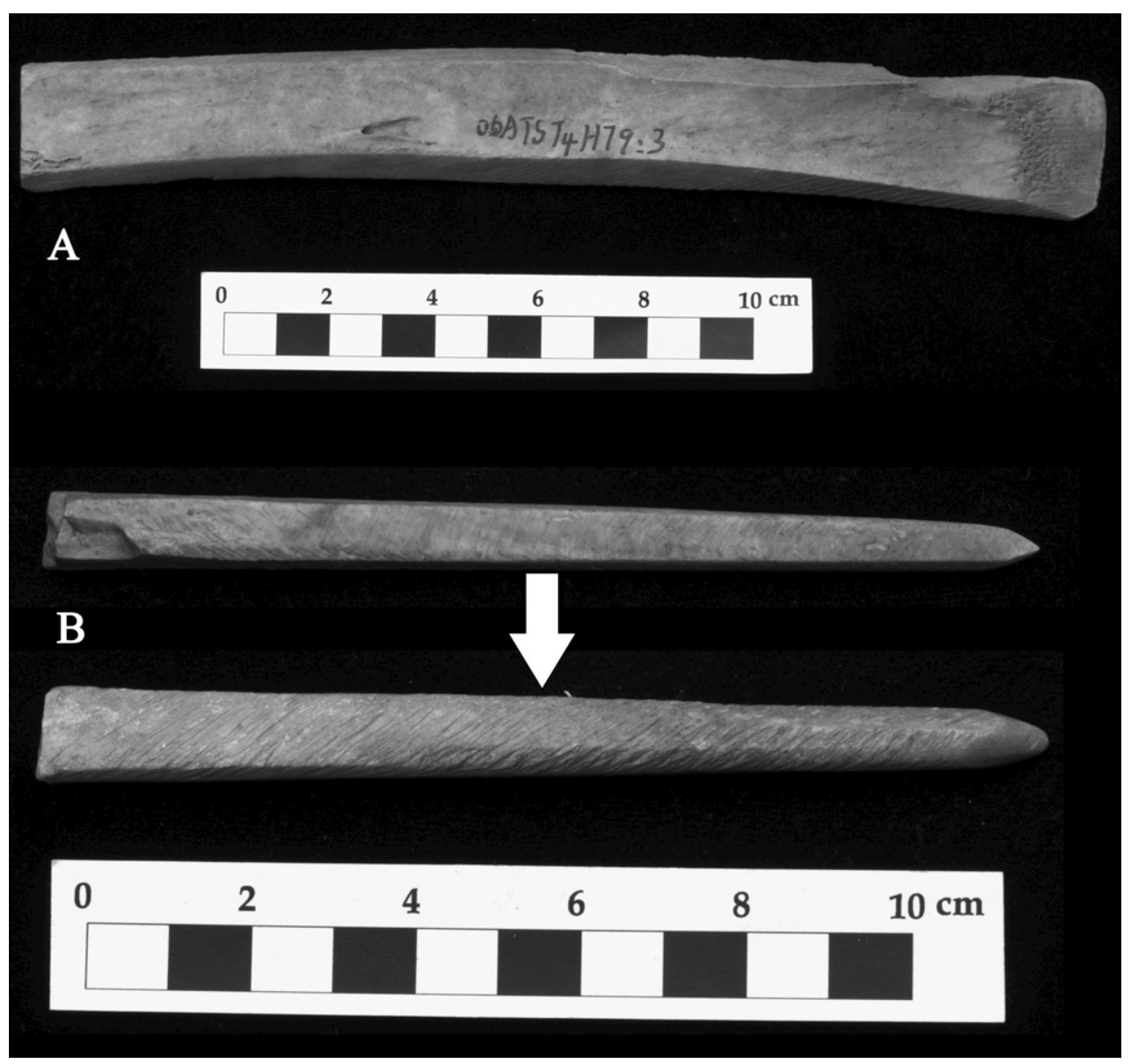

Fig. 17. Tiesanlu, Anyang bone tools: (A) large mammal semi-finished long-bone spatula [ATST4H79:3]; (B) antler awl sawed pre-form [ATST3H79:7-4]; (C) finished antler awl with rasp marks [ATST4H79:5-4].

reiterate, Group IV artifacts were either produced from large mammal long bones or antler, were completely modified from the original bone, and, where tool marks are visible, show the striations of saw cuts or the diagonal rasping of saw scraping-all exactly the same features of artifacts of the same type recovered from Tiesanlu. Beyond production method, the Guandimiao pins and arrowheads are formally, stylistically indistinguishable from those produced at Tiesanlu.

This formal match prompts us to further claim that the pins and arrowheads recovered at Guandimiao were not only the products of nonlocal, specialized workshops, but also that they almost certainly originated in the large-scale bone workshops of Anyang, perhaps Tiesanlu itself (Li et al. 2011). Our argument for the specific origin of the Guandimiao Group IV artifacts is strongest for the abstract design one-piece pins (Fig. 13, bottom row), a style of pin produced in large quantities at Tiesanlu, Anyang (Fig. 18). The basic production sequence for these pins is shown in Figure 18 (see also Campbell et al. 2011:1289, fig. 8). The articular ends of a large 

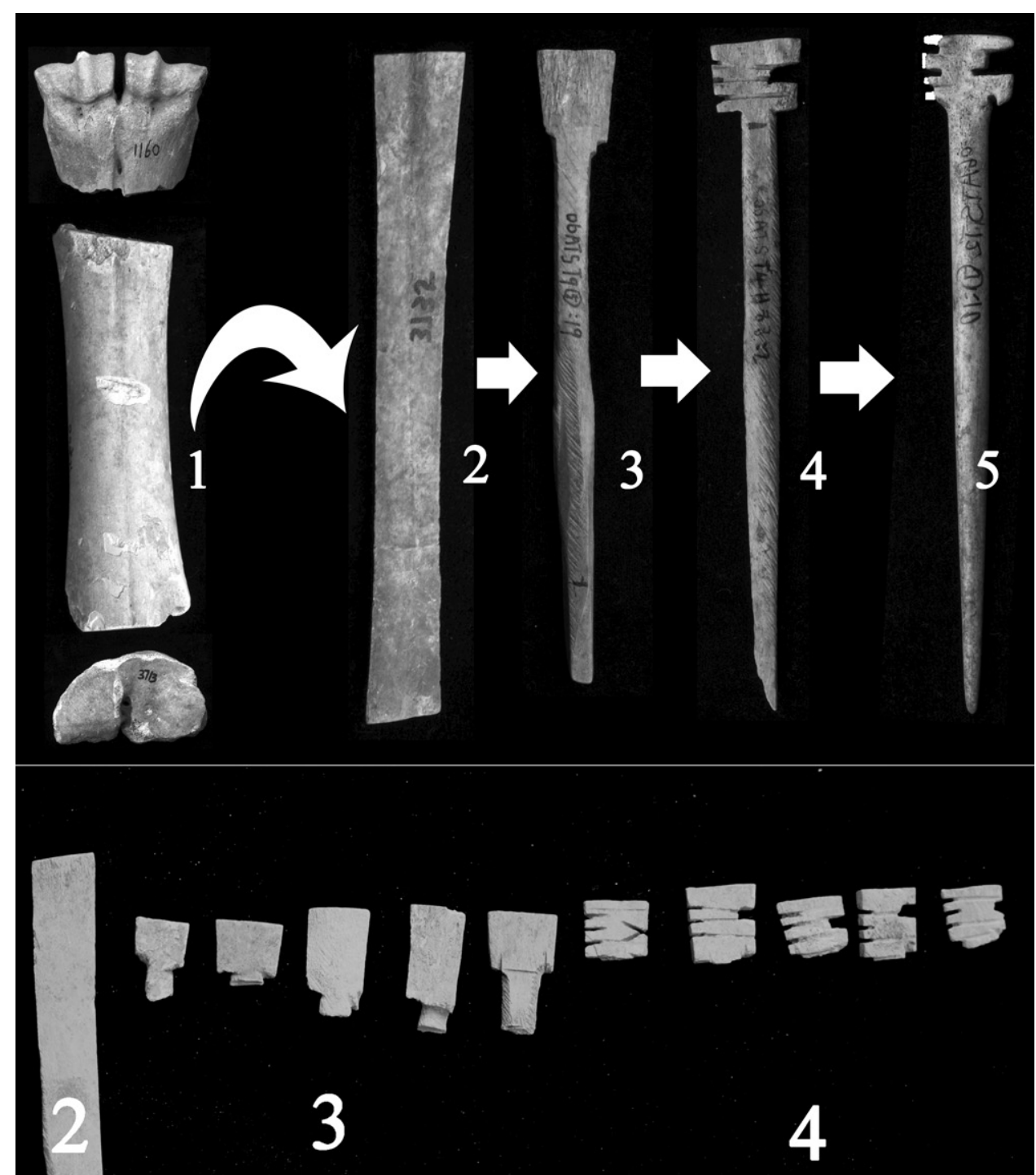

Fig. 18. Tiesanlu bone hairpin basic production sequence, steps 1-5 (top); abstract design one-piece pre-form and production wasters recovered from Tiesanlu, representing steps 2-4 (bottom). Note: the used pin on the top far right (step 5) was damaged, the white parts are reconstructions; this pin shows an overcut from step 3 when the pin body and head were delineated. 
mammal limb bone (most frequently cattle metapodials) are first removed with a saw (step 1). The resulting tube of bone is sawed into long strips of the maximum length and width of the finished hairpin (step 2). The basic shape is then delineated with a saw (step 3). The abstract design is subsequently executed on the head with a saw, and the edges of the pin body are scraped off with a diagonal motion of the saw (step 4). In the last stage, the pin body is polished smooth, as can be seen on a finished, used, and damaged pin recovered from Tiesanlu (step 5) (Fig. 18 top, far right pin). Each of these steps except the last was executed with a saw and, despite polishing, tool marks from the third and fourth steps frequently remain.

Such telltale production marks can be most easily be seen on half-finished pins such as those recovered from Tiesanlu (Fig. 18, Fig. 19A, B). The lack of the weathering and breakage usually accruing from a lifetime of use before an artifact enters the archaeological record allows us to see more clearly what the used pins in Figure 20 and Figure 13 originally looked like. In Figure 19, traces of the basic delineation of the pin shape can still be seen in the saw overcuts and undercuts at the intersection of the head and body. These traces and the last three stages of production are also obvious on most of the wasters in the bottom row of Figure 18 and the last three stages shown in the top row (especially the overcut on the used pin at the top far right of Fig. 18). Logically, a carefully produced saw cut demarcating a rectangular pinhead should be a right angle

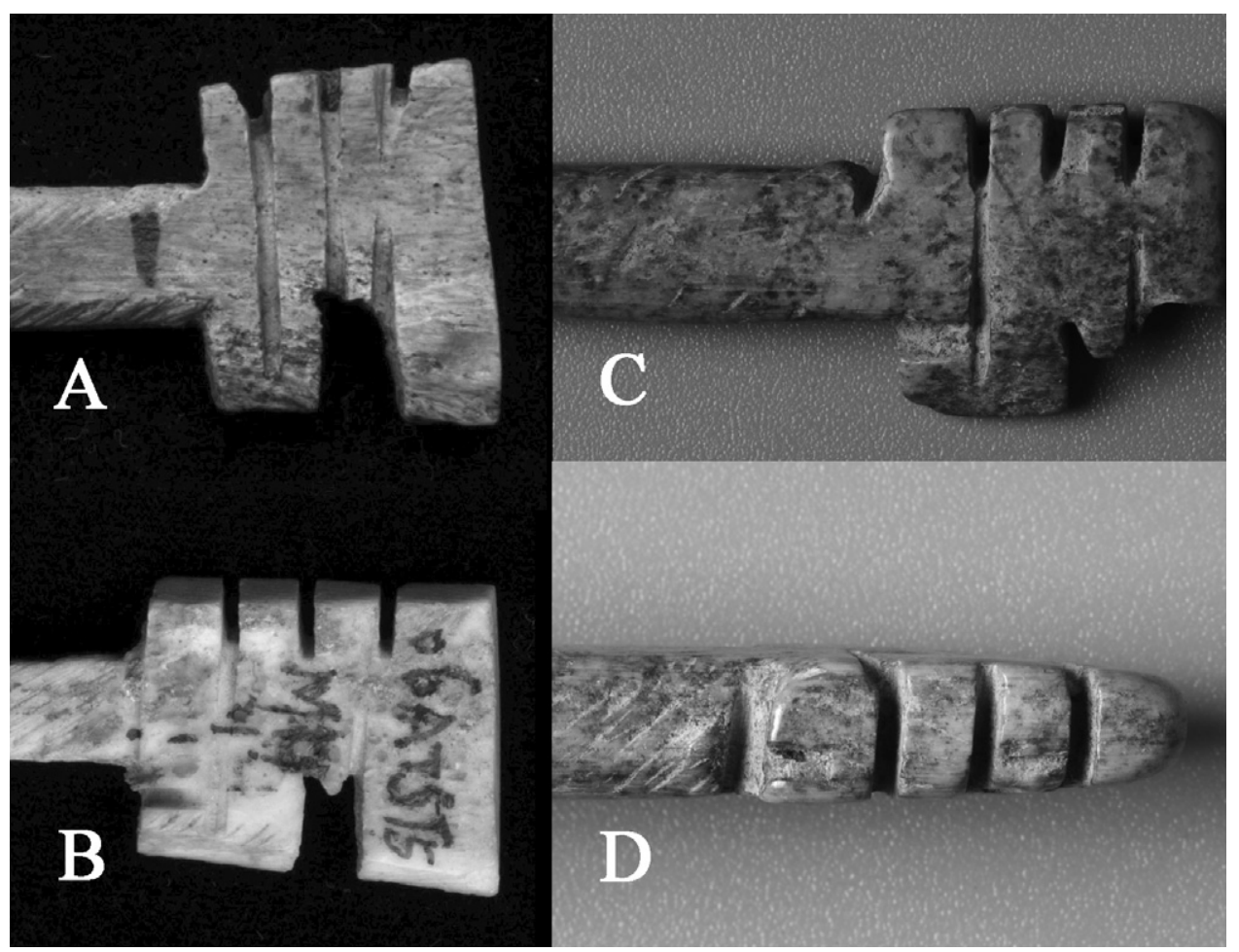

Fig. 19. Comparison of production marks on abstract design one-piece pins: (left) Tiesanlu, Anyang half-finished pins, (A) [ATST4H88: 2] and (B) [ATST5M10:1]; (right) Guandimiao used pin [HXYGT4111H1059(3):2], (C) front and (D) side. 
with perfectly straight edges; the semi-finished pin in Figure 19A comes closest to that ideal. The fact that this is frequently not the case even on used pins found at Tiesanlu (Fig. 20B) suggests that these pins were made in a simple and quickly executed manner and that producing imperfect angles or not-quite-straight edges was not enough reason for them to be discarded as wasters.

The execution of the design can be seen most clearly in Figure 19. The basic rectangular shape was cut with four saw cuts and then three parallel cuts were made on one side of the head; these were indifferently drawn across the surface to extend the design across the face of the pinhead. Then two more parallel saw cuts were made on the other side, the middle of which was snapped off to create, perhaps, the suggestion of a bird's head and feet. That the lines across the surface are of uneven depths and only sometimes cross the entire surface suggests these were single cuts with no retouching. The ragged edge of the breaks on the side demarcating the abstract "head" and "foot" likewise indicate a lack of finishing. The rasp marks on the body of many used examples of this kind of pin also indicate that, while polished smooth, the finishing stage was done relatively rapidly and carelessly. A composite picture of specialized mass production emerges when these lines of evidence are put together with the fact that the semi-finished pins in Figure 19A and 19B come from the large-scale bone workshop of Tiesanlu. Hundreds of wasters such as those seen in Figure 18 suggest that this style of pin was produced in the thousands if not tens of thousands at Tiesanlu. Overall estimates of this workshop suggest a total pin production in the millions, and ongoing research indicates high product specialization, division of labor, and divided production processes (Campbell et al. 2011; Li et al. 2011). Such bone pins were produced in vast quantities from abundant supplies of select raw materials, in standardized production steps, using specialized tools, and with a premium on production efficiency. These characteristics left such strong traces on the finished products that a lifetime of wear and tear could not erase them. The pin from Guandimiao in Figure 19C and 19D shows all of these signs: a serious overcut into the body, an overcut onto the head, an unevenly snapped space delimiting the "head" and "foot" of the abstract design, sawed surface lines of uneven depth, and rasp marks that

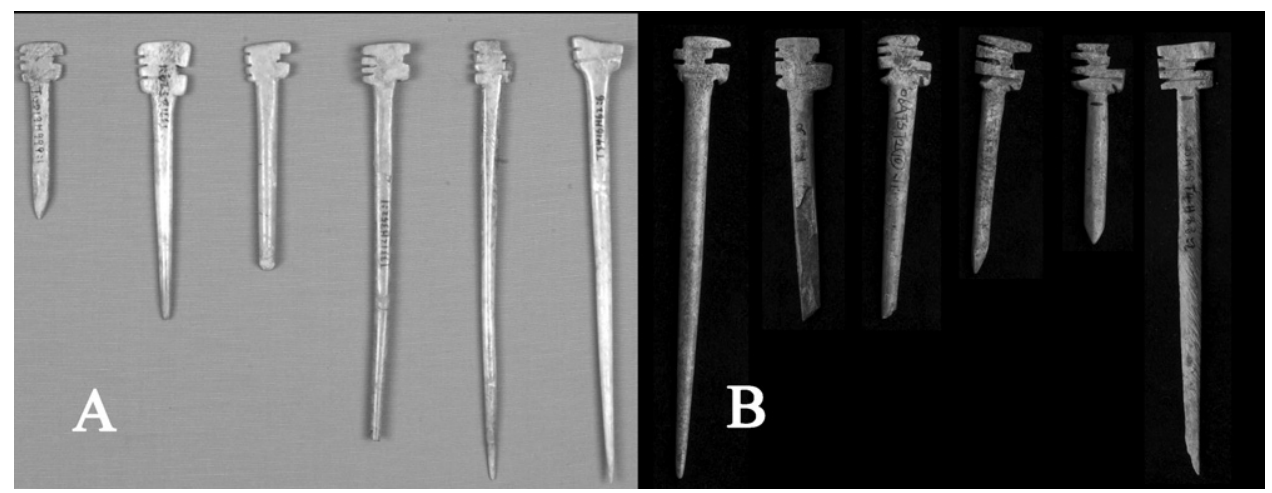

Fig. 20. Comparison of abstract design pins: (A) Guandimiao used pins; (B) Tiesanlu used pins (far right pin is half-finished). 
were not totally removed by polishing. Not only is this pin identical in style, tool marks, and apparent production steps to those produced at Tiesanlu, but also in its manner of execution - a manner consistent with mass production.

A comparison of used one-piece abstract design hairpins recovered from Guandimiao and Tiesanlu clearly show their identical form and style (Fig. 20). Aside from individually specific production errors, use-wear, and breakage before they entered the archaeological record, the pins are the same. Even the length of the unbroken or unreworked pins has been standardized. Indeed, if the backgrounds were the same or the pins mixed and artifact numbers erased, the two groups would be indistinguishable.

\section{CONCLUSION}

The Guandimiao worked bone assemblage has specific economic implications. Group I artifacts are expedient tools that were likely produced ad hoc whenever needed or as appropriate materials presented themselves. The Group II artifacts suggest relatively low-skill, low-labor, or semi-specialized local production perhaps at Guandimiao or perhaps at another nearby site with a part-time craftperson. The oracle-bones (Group III) are a different case; these highly specialized artifacts were carefully produced, employing a tool the marks of which are generally not seen on other bone artifacts from Guandimiao. The formal differences between Guandimiao and Anyang nonroyal, uninscribed oracle-bones and the presence of partially prepared oracle-bones at Guandimiao indicate that oracle-bone manufacture seems to have been the work of a local specialist.

Only Group IV suggests that Guandimiao was connected to wider economic networks. Formally distinguished from the other three groups by the choice of raw materials, production steps, tool marks, and degrees of skill, labor, and standardization, these artifacts (constituting over a quarter of the worked bone assemblage) were not produced locally. While the case for the plain polished hairpins and arrowheads is only based on formal distinction from the locally produced groups, we believe it is compelling. If bronze saws were locally available and local craftspeople were willing and able to cut large mammal long bones and antlers into pre-forms and shape and grind them as at Anyang, why do the locally produced tools at Guandimiao invariably lack the characteristic traces of such manufacture? Additionally, as the Tiesanlu site amply attests, the production of bone artifacts from large, dense mammal limb bones leaves a considerable amount of waste-conspicuously the articular ends of metapodials-among the best-preserving parts of bovid skeletons. At Anyang Tiesanlu, 34 metric tons of worked bone waste was recovered, including hundreds of half-finished wasters, but only a few dozen finished artifacts were found (Campbell et al. 2011). Guandimiao yielded a similar number of used bone artifacts from a comparable excavation area, but only a handful of fragments of expediently worked bone and no semi-finished artifacts. Clearly, Guandimiao was not a formal, specialized, and mass-producing bone-working site of the sort that leaves large quantities of debitage. Another indicator that Group IV artifacts were not produced at the site is that there is almost no evidence of large mammal long bone worked bone waste at Guandimiao; the one fragment found is very likely a later intrusion. 
If the hairpins and arrowheads were not locally made, then where were they produced? The decorated pins provide clues to the answer. As noted above, not only do they stylistically match those produced at Great Settlement Shang in large-scale bone workshops such as Tiesanlu, they bear the unmistakable signs of having been mass produced. While it is difficult to disprove the possibility of bone workshops in regional centers exactly replicating the capital workshops in both production method and artifact style, it does seem unlikely. The fact that no Anyang period large-scale bone-working sites have been discovered outside of Anyang to date (including at the best-known Anyang period secondary center of Daxingzhuang) further suggests an Anyang origin for these pins. (This assessment is based on coauthor Campbell's ongoing research on worked bone materials and finished artifacts at Daxingzhuang.) Given the amount of bone debitage created by large-scale specialized bone working of the kind that produced the decorated pins recovered from Guandimiao, the lack of discovery of such remains outside of Anyang during this period is unlikely to be simply a matter of bad luck.

More importantly than absence of evidence, however, is the positive evidence concerning the scale and nature of Anyang bone working. As some of us have argued elsewhere, millions of bone hairpins are estimated to have been produced at the Anyang bone workshops. Such high production likely outstripped the needs of Great Settlement Shang by a factor of three or four, raising the question of where the surplus went (Campbell et al. 2011; Li et al. 2011). The evidence from Guandimiao seems to provide a partial answer: surplus hairpins were exchanged far and wide, even to such tiny villages as Guandimiao some $200 \mathrm{~km}$ distant from Anyang. The concentration of bone workshops at Anyang is likely explained by the ready availability of massive quantities of cattle bone and antler, bronze tools, skilled craftspeople, and the efficiency of economies of scale.

While the concentration of kilns and pottery production waste at the Anyang period village of Guandimiao indicates remarkable intraregional economic specialization, the bone assemblage reveals evidence of a hitherto unsuspected degree of integration into the larger economic networks of Great Settlement Shang. In addition, while nearly one-third of the non-oracular bone artifact assemblage was of the mass-produced bone workshop type, these artifacts were discovered scattered across the site in house floors, wells, or deposits between features as well as in middens. This suggests that the entry of these artifacts into the archaeological record was haphazard and perhaps largely unintentional. Moreover, given the evidence of the curation and reuse of even the shortest pins, they seem not to have been discarded lightly. If this is so, then the 84 nonoracular bone artifacts recovered from the site likely represent a tiny fraction of the artifacts used by generations of villagers at Guandimiao. Considering the small size of the site and its population, this in turn means that, despite the relatively small absolute numbers of recovered bone artifacts that can be linked to Anyang bone working, the original scale of the exchange would have been fairly significant (Li et al. forthcoming) and probably more regular than occasional gift exchange or down-the-line trading (Renfrew 1975). Understanding the nature of this exchange and the Shang political economy more generally will have to wait for future research; but at this point, it is already clear that there was far more economic specialization and integration both intra- and inter-regionally in the Central Plains during the Anyang period than was previously suspected. 


\section{REFERENCES CITED}

Brumfiel, Elizabeth M., and Timothy K. Earle

1987 Specialization, exchange, and complex societies: An introduction, in Specialization, Exchange, and Complex Societies: 1-9, ed. E. M. Brumfiel and T. K. Earle. Cambridge: Cambridge University Press.

CAmpbell, Roderick

2009 Towards a networks and boundaries approach to early complex polities: The late Shang case. Current Anthropology 50(6):821-848.

2014 Archaeology of the Chinese Bronze Age: From Erlitou to Anyang. Los Angeles: Cotsen Institute of Archaeology Press.

2018 Violence, Kinship and the Early Chinese State: The Shang and their World. Cambridge: Cambridge University Press.

Campbell, Roderick, Zhipeng Li, Yuling He, and Yuan Jing

2011 Consumption, exchange and production at the Great Settlement Shang: Bone-working at Tiesanlu, Anyang. Antiquity 85:1279-1297.

ChANG, K. C.

1975 Ancient trade as economics or as ecology, in Ancient Civilization and Trade: 211-224, ed. J. Sabloff and C. C. Lamberg-Karlovsky. Albuquerque: SAR, University of New Mexico Press.

1980 Shang Civilization. New Haven, CT: Yale University Press.

1989 Ancient China and its anthropological significance, in Archaeological Thought in America: 155-166, ed. C. C. Lamberg-Karlovsky. Cambridge: Cambridge University Press.

Choyke, Alice

1997 The manufacturing continuum. Anthropozooalogica 25-26:65-72.

2013 Hidden agendas: Ancient raw material choice for worked osseous objects in Central Europe and beyond, in From these Bare Bones: Raw Materials and the Study of Worked Osseous Objects: 1-11, ed. A. Choyke and S. O'Connor. Oxford: Oxbow Books.

Costin, C.

1991 Craft specialization: Issues in defining, documenting, and explaining the organization of production, in: Archaeological Method and Theory, vol. 3: 1-56, ed. M. B. Schiffer. Tucson: University of Arizona Press.

FLAD, ROWAN

2007 Rethinking the context of production through the archaeological study of ancient salt production in the Sichuan Basin, China, in Rethinking Craft Specialization in Complex Societies: Archaeological Analyses of the Social Meaning of Production: 108-128, ed. Z. Hruby and R. Flad. Arlington, VA: Archaeological Papers of the American Anthropological Association.

2008 Divination and power: A multi-regional view of the development of oracle-bone divination in Early China. Current Anthropology 49(3):403-437.

Greene, Alan, and Ian Lindsay

2012 Mobility, Territorial Commitments, and Political Organization among Late Bronze Age Polities in Southern Caucasia. Archeological Papers of the American Anthropological Association 22:54-71.

Henan Sheng Wenwu KaOgu YanjIUSUO 河南省文物考古研究所

2008 Henan Xingyangshi Guandimiao yizhi Shangdai wanqi yicun fajue jianbao 河南䓠阳市关帝 庙遗址商代晚期遗存发掘简报 [Preliminary excavation report for the remains of the Late Shang site of Guandimiao, Xingyang municipality, Henan province]. Kaogu 7:32-46.

2009 Henan Xingyangshi Guandimiao yizhi kaogu faxian yu renshi 河南水阳市关帝庙遗址考古 发现与认识 [Archaeological discovery and understanding of the Guandimiao site, Xingyang municipality, Henan province]. Huaxia Kaogu 3:8-13.

INOMATA, TAKESHI

2001 The power and ideology of artistic creation: Elite craft specialists in classic Maya society. Current Anthropology 42(3):321-349.

Li Suting, Roderick Campbell, and Hou Yanfeng

forthcoming Guandimiao: A Shang village and its significance. Antiquity.

Li YUNG-TI

2003 The Anyang bronze foundaries: Archaeological remains, casting technology and production remains. Ph.D. diss. Anthropology Department, Harvard University. 
Li Zhipeng 李志鹏, He Yuling 何毓灵, AND JiAng Yude 江雨德 [Roderick CAmpbell]

2011 Yinxu Wan Shang zhigu zuofang yu zhigu shougongye de yanjiu huigu yu tantao 殷墟晚商制 骨作坊与制骨手工业的研究回顾与探讨 [Research on Late Shang bone workshops and the bone working industry at Yinxu]. Sandai Kaogu 4:471-486.

MacGregor, Arthur

1985 Bone, Ivory and Horn. The Technology of Skeletal Material Since the Roman Period. London: Croom Helm.

MacGregor, Arthur G., And J. D. Currey

1983 Mechanical properties as conditioning factors in the bone and antler industry of the 3rd to 13th century AD. Journal of Archaeological Science 10:71-77.

RenFrew, Colin

1975 Trade as action at a distance: Questions of integration and communication, in Ancient Civilization and Trade: 3-60, ed. J. Sabloff and C. C. Lamberg-Karlovsky. Albuquerque: SAR, University of New Mexico Press.

Rice, Prudence M.

1981 Evolution of specialized pottery production: A trial model. Current Anthropology 22:219-240.

SHelach-Lavi, GideON

2015 The Archaeology of Early China: From Prehistory to the Han Dynasty. Cambridge: Cambridge University Press.

Sinopoli, Carla

2003 The Political Economy of Craft Production: Crafting Empire in South India. Cambridge: Cambridge University Press.

Underhill, ANN, AND FANG HuI

2004 Early state economic systems in China, in Archaeological Perspectives on Political Economies: 129-144, ed. G. Feinman and L. Nicholas. Salt Lake City: University of Utah Press.

YANG SHENGNAN 杨升南 AND MA JIFAN 马季凡

2010 Shang dai jingji yu keji 商代经济与科技 [Shang Dynasty Economy and Technology], Shang dai shi - juan liu [vol. 6 of The History of the Shang Dynasty]. Beijing: Zhongguo shehuikexue chubanshe.

YuAN, Jing, AND ROWAN FLAD

2005 New zooarchaeological evidence for changes in Shang dynasty animal sacrifice. Journal of Anthropological Archaeology 24:252-270.

ZHONGGUO SHEHUI KEXUE YUAN, KAOGU YANIU SUO 中国社会科学院考古研究所

2003 Zhongguo kaoguxue: Xia, Shang juan 中国考古学: 夏商卷 (Chinese Archaeology: Xia and Shang). Beijing: Zhongguo shehuikexue chubanshe.

2015 Anyang Yinxu Tiesanlu zhiguzuofang yizhi fajue jianbao 安阳殷墟铁三路制骨作坊遗址发 掘简报 [Preliminary excavation report of the bone working site at Tiesanlu, Yinxu, Anyang]. Kaogu (8):37-62. 